This article has been accepted for publication in MNRAS (c) 2017 The Authors. Published by Oxford University Press on behalf of the Royal Astronomical Society. All rights reserved. 


\title{
A methodology to select galaxies just after the quenching of star formation
}

\author{
Annalisa Citro, ${ }^{1,2 \star}$ Lucia Pozzetti, ${ }^{2 \star}$ Salvatore Quai, ${ }^{1,2}$ Michele Moresco, ${ }^{1,2}$ \\ Livia Vallini ${ }^{1,2,3}$ and Andrea Cimatti ${ }^{1,4}$ \\ ${ }^{1}$ Dipartimento di Fisica e Astronomia, Università di Bologna, Via Gobetti 93/2, I-40129, Bologna, Italy \\ ${ }^{2}$ INAF-Osservatorio Astronomico di Bologna, Via Gobetti 93/3, I-40129, Bologna, Italy \\ ${ }^{3}$ Nordita, KTH Royal Institute of Technology and Stockholm University, Roslagstullsbacken 23, SE-106 91 Stockholm, Sweden \\ ${ }^{4}$ INAF-Osservatorio Astrofisico di Arcetri, Largo E. Fermi 5, I-50125, Firenze, Italy
}

Accepted 2017 April 12. Received 2017 April 12; in original form 2016 December 22

\begin{abstract}
We propose a new methodology aimed at finding star-forming galaxies in the phase which immediately follows the star-formation (SF) quenching, based on the use of high- to lowionization emission line ratios. These ratios rapidly disappear after the SF halt, due to the softening of the UV ionizing radiation. We focus on [O III] $\lambda 5007 / \mathrm{H} \alpha$ and $[\mathrm{Ne}$ III] $\lambda 3869 /[\mathrm{O}$ II] $\lambda 3727$, studying them with simulations obtained with the CLOuDY photoionization code. If a sharp quenching is assumed, we find that the two ratios are very sensitive tracers as they drop by a factor of $\sim 10$ within $\sim 10 \mathrm{Myr}$ from the interruption of the SF; instead, if a smoother and slower SF decline is assumed (i.e. an exponentially declining SF history with $e$-folding time $\tau=200 \mathrm{Myr}$ ), they decrease by a factor of $\sim 2$ within $\sim 80$ Myr. We mitigate the ionizationmetallicity degeneracy affecting our methodology using pairs of emission line ratios separately related to metallicity and ionization, adopting the $[\mathrm{N}$ II] $\lambda 6584 /[\mathrm{O}$ II] $\lambda 3727$ ratio as metallicity diagnostic. Using a Sloan Digital Sky Survey galaxy sample, we identify 10 examples among the most extreme quenching candidates within the [O $\mathrm{III}] \lambda 5007 / \mathrm{H} \alpha$ versus $[\mathrm{N} \mathrm{II]} \lambda 6584 /$ [O II] $\lambda 3727$ plane, characterized by low [O III] $\lambda 5007 / \mathrm{H} \alpha$, faint [Ne III] $\lambda 3869$, and by blue dust-corrected spectra and $(u-r)$ colours, as expected if the SF quenching has occurred in the very recent past. Our results also suggest that the observed fractions of quenching candidates can be used to constrain the quenching mechanism at work and its time-scales.
\end{abstract}

Key words: ISM: H II regions - ISM: lines and bands - galaxies: evolution-galaxies: ISM.

\section{INTRODUCTION}

One of the key questions in the current studies of galaxy evolution is to understand the origin of the dichotomy which segregates galaxies into the blue cloud (i.e. late-type, star-forming and disc-dominated galaxies) and the red sequence (i.e. early-type, quiescent and bulgedominated galaxies). For $M>10^{10} \mathrm{M}_{\odot}$, this bimodality is clearly visible both at $z \sim 0$ (Strateva et al. 2001; Blanton et al. 2003; Kauffmann et al. 2003a; Baldry et al. 2004) and at higher redshifts (Bell et al. 2004, 2012; Willmer et al. 2006; Cassata et al. 2008; Brammer et al. 2009; Whitaker et al. 2011; Wuyts et al. 2011; Cheung et al. 2012; Muzzin et al. 2013) and concerns both galaxy colours and structure (McIntosh et al. 2014).

There is a general consensus that the main process underlying the galaxy bimodality is the build-up of the red sequence population at $M<10^{11} \mathrm{M}_{\odot}$, driven by the quenching of the star formation (SF) and by a morphological transformation, which turns late-type into

^E-mail: annalisa.citro@unibo.it (AC); lucia.pozzetti@oabo.inaf.it (LP) early-type morphologies (e.g. Drory et al. 2004; Faber et al. 2005; Pozzetti et al. 2010).

Still unconstrained are the mechanisms that are able to modify both SF and morphology and their corresponding time-scales. Several hypotheses have been proposed to explain the quenching of the SF in blue galaxies, such as gas stripping (e.g. Gunn \& Gott 1972), morphological or gravitational quenching (Martig et al. 2009; Genzel et al. 2014), shock heating of infalling cold gas by the hot halo (Dekel \& Birnboim 2006) or an exhaustion of the gas supply (e.g. Larson, Tinsley \& Caldwell 1980). Moreover, in massive galaxies, the role of AGNs in influencing galaxy evolution and quenching SF is supported by several observations (Hopkins et al. 2005; Kaviraj et al. 2007; Diamond-Stanic et al. 2012; Fabian 2012, and references therein, Cimatti et al. 2013; Cicone et al. 2014; Förster Schreiber et al. 2014) and corroborated by the theoretical results obtained combining $N$-body simulations of dark matter haloes evolution (Springel et al. 2005; Boylan-Kolchin et al. 2009) with semi-analytic models for galaxy formation (White \& Frenk 1991; Springel et al. 2005; Lu et al. 2011; Benson 2012). However, other models are capable of forming rapidly quiescent galaxies without 
invoking the AGN feedback (e.g. Khochfar \& Silk 2006; Naab, Khochfar \& Burkert 2006, 2009; Johansson, Thomas \& Maraston 2012). Stellar or supernova (SN) feedback is most likely channel for the SF quenching in low-mass galaxies (e.g. $<10^{10} \mathrm{M}_{\odot}$; Kaviraj et al. 2007). Several mechanisms have also been invoked to explain the morphological transformation. Numerical simulations have shown that major merging can give rise to elliptical and S0 galaxies (Bekki 1998) and that also minor merging can play an important role in spheroid and bulge growth (Bournaud, Jog \& Combes 2007; Naab et al. 2007). From an observational point of view, evidence that the morphological transformation can also be induced by environmental mechanisms (Larson et al. 1980; Farouki \& Shapiro 1981; Moore et al. 1999; Quilis, Moore \& Bower 2000; ; Bekki, Couch \& Shioya 2002) or by the secular growth of pseudobulges (Courteau, de Jong \& Broeils 1996; Norman, Sellwood \& Hasan 1996; MacArthur, Courteau \& Holtzman 2003; Kormendy \& Kennicutt 2004; Debattista et al. 2006) has been found.

Having intermediate colours between the blue cloud and the red sequence, galaxies populating the so-called green-valley (e.g. Salim 2014; Schawinski et al. 2014) are generally considered as the transiting objects par excellence (Martin et al. 2007; Mendel et al. 2013; Salim 2014; Schawinski et al. 2014). Among these, the most interesting population certainly consists of those galaxies which have just entered the quenching phase (within a few Myr). Although hampered by the short duration of the quenching process, the search for galaxies in this critical phase of evolution has been carried on by several authors in the past decades. Galaxies characterized by both a tidally disturbed morphology and intermediate colours (e.g. Schweizer \& Seitzer 1992; Tal et al. 2009) or low level of recent SF (Kaviraj 2010), young elliptical galaxies (Sanders et al. 1988; Genzel et al. 2001; Dasyra et al. 2006) and very recent post-merger remnants with strong morphological disturbances (Hibbard \& van Gorkom 1996; Rothberg \& Joseph 2004; Carpineti et al. 2012) have been considered as valid 'recent time' quenching candidates. Moreover, many attempts aimed at spectroscopically identifying quenching galaxies come from the investigations of the post-starburst $(\mathrm{E}+\mathrm{A}$ or K+A) galaxies' UV and optical spectra, whose strong Balmer absorption lines and missing [O II] $\lambda 3727$ (hereafter [O II]) and $\mathrm{H} \alpha$ emission lines (Couch \& Sharples 1987; Poggianti et al. 2004; Quintero et al. 2004; Balogh et al. 2011; Muzzin et al. 2012; Mok et al. 2013; Wu et al. 2014) have been interpreted as signs of a recent halt of the SF (Dressler \& Gunn 1983; Zabludoff et al. 1996; Quintero et al. 2004; Poggianti et al. 2008; Wild et al. 2009). The scarcity of galaxies that are in the transition phase suggests that, whatever mechanism may be responsible for the SF shut-off, it has to happen on short time-scales (Tinker, Wechsler \& Zheng 2010; Salim 2014). The relatively short duration of the quenching process is also suggested by the surprising identification of a significant number of galaxies that look already quiescent at $z \sim 4-5$, when the Universe was only 1-1.5 Gyr old (e.g. Mobasher et al. 2005; Wiklind et al. 2008; Juarez et al. 2009; Brammer et al. 2011; Marsan et al. 2015; Citro et al. 2016). However, a general and coherent picture concerning the SF quenching is still lacking. Very recently, evidence that the quenching of the SF could be a separated process with respect to the morphological transformation has come from the photometric and spectroscopic investigations of passive spiral galaxies (Fraser-McKelvie et al. 2016).

Further uncertainties concern the typical time-scales of the quenching process, which are far to be constrained. For instance, galaxies with early-type morphologies seem to be characterized by shorter quenching time-scales, with respect to galaxies with latetype morphologies (Schawinski et al. 2014), and the time-scales derived observationally are generally shorter than the ones derived using $N$-body simulations and semi-analytic models (Muzzin et al. 2012; Mok et al. 2013; Wetzel et al. 2013; Taranu et al. 2014).

Emission line ratios have been proved to be powerful tools to constrain the ionization state and the properties of galaxies both at $z \sim 0$ and at higher redshift (e.g. Dopita et al. 2000, 2006; Kewley et al. 2001, hereafter Kew01; Levesque, Kewley \& Larson 2010, hereafter Lev10; Kewley et al. 2013; Kashino et al. 2016), but this kind of studies is always hampered by the degeneracies affecting spectra and emission lines, which in many cases makes it difficult to disentangle what is the origin of the emission line ratio intensity (e.g. Dopita et al. 2006). However, a general view on how the emission line ratios can help in tracing the quenching phase is lacking, especially when the very early epochs after the quenching are involved.

In this work, we propose a methodology aimed at identifying galaxies in the phase which immediately follows the quenching of the SF, based on the search for galaxies with weak high-ionization and strong low-ionization emission lines in their spectra. In particular, we adopt the $[\mathrm{O}$ III] $\lambda 5007$ (hereafter [O III]) and $[\mathrm{Ne}$ III] $\lambda 3869$ (hereafter [ $\mathrm{Ne}$ III]) high-ionization lines, and the $\mathrm{H} \alpha$ and [O II] $\lambda 3727$ (hereafter [O $\mathrm{OI}]$ ) low-ionization lines. [O III] and [ $\mathrm{Ne}$ III] probe the presence of short-lived and very massive $\mathrm{O}$ stars able to provide photons hard enough to doubly ionize oxygen and neon; $\mathrm{H} \alpha$ and [O II], being excited by softer photons, probe the presence of colder $\mathrm{O}$ stars and B stars.

We simulate the emission line ratios using the photoionization code CLOUDY (Ferland et al. 1998), assuming short time-scales for the quenching. We also combine the emission line ratios with metallicity diagnostics, in order to mitigate the ionization-metallicity degeneracy.

\section{THE PROPOSED APPROACH}

We propose a methodology able to identify galaxies in the phase just after the quenching of the SF, based on emission line ratios. If the SF is quenched in a star-forming region, the number of UV hydrogen ionizing photons (i.e. $\lambda<912 \AA$ ) provided by the central ionizing source declines. In particular, since massive $\mathrm{O}$ stars are the first to disappear, the harder UV photons are the first to drop, leading to a softening of the UV ionizing spectrum. As a consequence, high-ionization lines, which can be excited only by the hardest ionizing photons, rapidly disappear; low-ionization lines, which can be produced also by colder $\mathrm{O}$ stars and B stars, remain strong at later times.

This allows us to devise a new methodology for identifying quenching galaxies. We search for galaxy spectra characterized by very faint high-ionization lines, like [O III] and [ $\mathrm{Ne}$ III], but still strong low-ionization lines, like $\mathrm{H} \alpha$ and [O II]. We put into practice our methodology by looking for galaxy spectra with high signal-tonoise ratio $(\mathrm{S} / \mathrm{N}) \mathrm{H} \alpha$ emission but very low values of the $\left[\mathrm{O}_{\mathrm{III}}\right] / \mathrm{H} \alpha$ and the $[\mathrm{Ne}$ III]/[O II] emission line ratios. These lines are observable in a wide range of redshifts (e.g. $0<z<2$ ) with optical and nearinfrared spectroscopy. In particular, [Ne III]/[O II], despite involving a very faint emission line like [ $\mathrm{Ne}$ III] $\lambda 3869$ (hereafter [ $\mathrm{Ne}$ III]), has the advantage of being basically unaffected by dust extinction, including emission lines which are very close in wavelength. We also investigated the possibility of using a line ratio less affected by dust extinction correction such as the $[\mathrm{O}$ III] $\lambda 5007 / \mathrm{H} \beta$ (hereafter $\left[\mathrm{O}_{\mathrm{III}}\right] / \mathrm{H} \beta$ ), which has also the advantage of involving stronger emission lines than $[\mathrm{Ne} \mathrm{III}] /[\mathrm{O}$ II] 
In the following, we assume a sharp quenching of the SF as extreme case. Moreover, we illustrate the results obtained if a more realistic, smoother but still short decline of the SF is adopted. This is in agreement with recent studies arguing that quenching processes occurring on 100-200 Myr time-scales can be modelled by a sudden interruption of the SF (e.g. Schawinski et al. 2014; Ciesla et al. 2016; Schaefer et al. 2017).

\section{MODELLING THE QUENCHING PHASE}

In order to investigate the behaviour of the proposed emission line ratios during the quenching phase, we simulate star-forming regions until their quiescent phase. Here we describe the main ingredients of our photoionization models.

We consider a star-forming region as formed by a central source of energy, with a given spectral energy distribution (SED) and intensity, surrounded by a spherical cloud. We simulate its final spectrum by means of the photoionization code CLOUDY (version 13.03, Ferland et al. 1998; Ferland et al. 2013), adopting a plane-parallel geometry (i.e. the simplest geometry allowed by the code), in which the thickness of the photoionized nebula is very small compared to the distance to the photoionizing source.

The shape of the ionizing source is simulated by means of different stellar synthetic spectra, such as the Leitherer et al. (1999) (STARBURST99) and Bruzual \& Charlot (2003) (BC03) models.

The adopted STARBURST 99 synthetic spectra are simple stellar populations (SSPs) with a fixed mass of $M=10^{6} \mathrm{M}_{\odot}$, metallicities $Z=0.004,0.008,0.02,0.04$ and a Salpeter initial mass function (IMF) (with $m_{\text {low }}=1$ and $m_{\text {up }}=100 \mathrm{M}_{\odot}$ ). They are computed using Lejeune-Schmutz stellar atmospheres (Schmutz, Leitherer \& Gruenwald 1992; Lejeune, Cuisinier \& Buser 1997) and Geneva-HIGH 1994 evolutionary tracks (Meynet et al. 1994; Leitherer et al. 1999). This set of ingredients is in agreement with the ones generally used in the literature (e.g. Kew01; Dopita et al. 2006; Lev10).

For BC03 synthetic spectra, we adopt SSPs with metallicities $Z$ $=0.004,0.008,0.02$ and 0.04. In particular, since the default BC03 highest metallicity is $Z=0.05$, we interpolate the metallicities to create $\mathrm{BC} 03$ models with $Z=0.04$, in order to be consistent with the STARBURST 99 results. These models are normalized to $M=1 \mathrm{M}_{\odot}$ and assume a Chabrier IMF with $m_{\text {low }}=0.1$ and $m_{\text {up }}=100 \mathrm{M}_{\odot}$. The slope of these two IMFs differs at $M<1 \mathrm{M}_{\odot}$, but we expect this difference not to influence the shape of the final SED, since very low mass stars contribute mostly to the mass of a stellar population rather than to its UV spectral properties. Moreover, since we are interested in emission line ratios, we can neglect the different mass normalization of the two models.

The intensity of the ionizing source is parametrized by the adimensional ionization parameter $U$, which is defined as the ratio between the mean intensity of the radiation field and the density of the ionized gas. In the plane-parallel case, $U$ can be written as (Tielens 2010):

$U=F_{0} / n_{\mathrm{H}} c$,

where $n_{\mathrm{H}}$ is the hydrogen number density of the photoionized gas, $c$ is the speed of light and $F_{0}$ is the flux of the UV ionizing photons $(\lambda<912 \AA)$ striking the photoionized cloud. $F_{0}$ is proportional to the number of UV hydrogen ionizing photons $Q(H)$, which in turn depends on the stellar metallicity, the stellar mass, the starformation rate (SFR), the age and the IMF of the ionizing central source. For instance, higher masses, higher SFRs, younger ages or top-heavy IMFs, which all imply a higher number of massive stars, lead $Q(H)$ to increase. Moreover, $F_{0}$ depends on the proximity of the

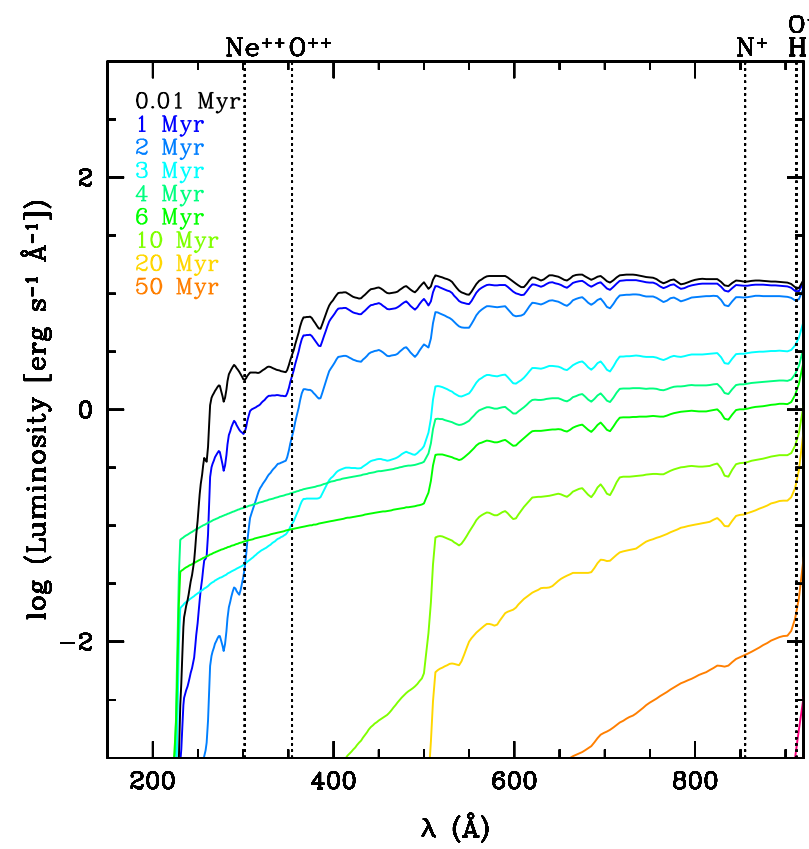

Figure 1. Time evolution of a STARBURST99 SSP SED with solar metallicity (spectra get older from blue to orange, as reported in the top left of the figure). Black dotted vertical lines indicate the wavelengths corresponding to the ionization energies of the emission lines analysed in this work, as indicated.

central stars to the photoionized nebula. As explained in Section 2, when a galaxy quenches its SF, $Q(H)$ declines due to the aging and the softening of the ionizing SED. As a consequence, $F_{0}$ decreases and so does the ionization parameter.

Throughout this work, we adopt two kinds of models, accounting for the decrease of the ionization parameter and the decline of the number of ionizing photons due to the quenching process.

(i) Fixed-age models. To fit with the majority of the literature studies (e.g. Dopita et al. 2006; Lev10; Kashino et al. 2016), we assume the central source to be an SSP with a given metallicity $(Z=$ $0.004,0.008,0.02$ and 0.04 ) and a fixed age of 0.01 Myr. Moreover, we adopt a grid of decreasing ionization parameters in order to simulate different ionization levels. We assume a grid of fixed-age ionization parameters $\log (U)_{0}$ going from -3.6 to -2.5 with steps of 0.1 dex, to be consistent with the observations of unresolved starforming $\mathrm{H}$ II regions $(\log (U) \lesssim-2.3$, see Yeh \& Matzner 2012), local $\mathrm{H}_{\text {II }}$ regions $(-3.2<\log (U)<-2.9$, see Dopita et al. 2000) and star-forming galaxies (see Moustakas, Kennicutt \& Tremonti 2006; Moustakas et al. 2010). In these models, given a metallicity, the shape of the ionizing source is fixed, regardless of the ionization parameter, and models with the lowest $\log (U)_{0}$ can describe starforming regions, but with very low densities of ionizing photons.

(ii) Evolving-age models. We also construct models which take account of the shape variation of the ionizing SED as a function of time after SF is stopped. This evolution is illustrated in Fig. 1 for an SSP of solar metallicity. The UV ionizing flux decreases as a function of time, with harder energies disappearing first, due to the sudden disappearance of the most massive $\mathrm{O}$ stars able to produce them. This behaviour is also visible in Fig. 2, which shows the time evolution of the number of ionizing photons below different energy thresholds (i.e. the ones relative to the emission lines studied in this work: $\mathrm{H}$ and $\mathrm{O}^{+}, 13.6 \mathrm{eV} ; \mathrm{O}^{++}, 35 \mathrm{eV}$ and $\mathrm{Ne}^{++}, 41 \mathrm{eV}$ ) and for different metallicities. The number of ionizing photons decreases 


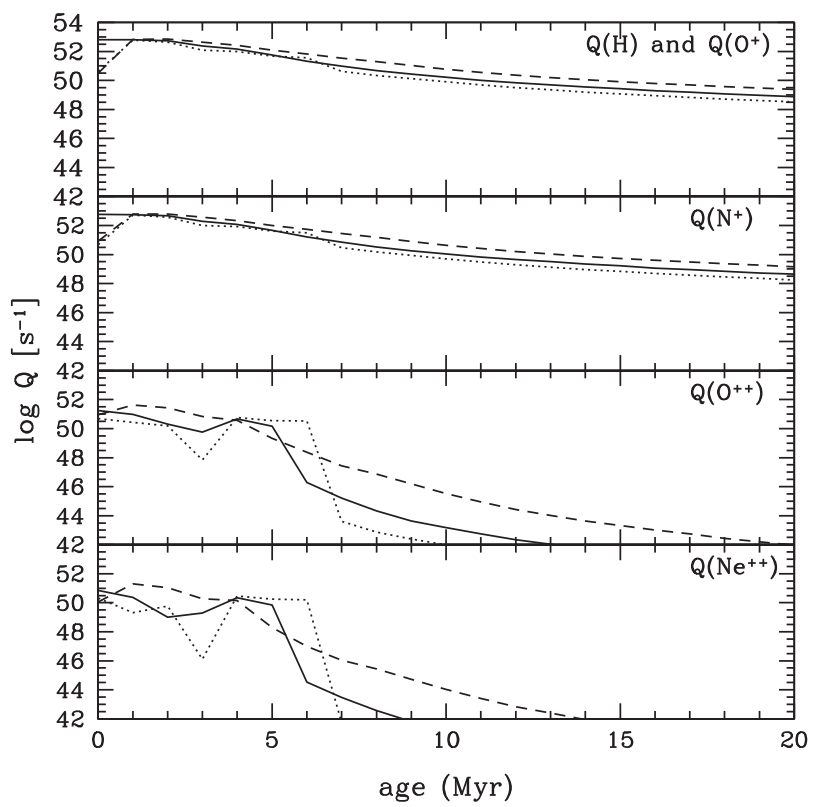

Figure 2. Time evolution of the number of photons able to ionize $\mathrm{H}$ and $\mathrm{O}^{+}$, $\mathrm{N}^{+}, \mathrm{O}^{++}$and $\mathrm{Ne}^{++}$, from top to bottom. Note that $\mathrm{H}$ and $\mathrm{O}^{+}$have the same ionization potential (i.e. $13.6 \mathrm{eV}$ ) and thus are illustrated within the same panel. Curves are relative to a STARBURST99 SSP with $\log \left(\mathrm{M} / \mathrm{M}_{\odot}\right)=10^{6}$ and three different metallicities $(Z=0.004$, dashed; $Z=0.02$, solid; $Z=$ 0.04 , dotted).

with time, and its decline is more pronounced for harder energies and higher metallicities. Moreover, the effect of the increased metallicity is more visible at the harder energies, which are more absorbed due to the larger stellar opacities. To account for the softening of the UV ionizing spectrum as a function of time after the SF quenching, in these models we simulate the central ionizing source using an SSP with given metallicity $(Z=0.004,0.008,0.02$ and 0.04$)$ and age going from 0.01 to $10 \mathrm{Myr}$. In particular, the youngest SSP of $0.01 \mathrm{Myr}$ is taken as a representative of a still star-forming region (this kind of assumption is often used in the literature, e.g. Kew01; Dopita et al. 2006; Lev10), while older SSPs are used to describe the epochs subsequent to the SF quenching. The still star-forming region can have an ionization state described by one of the fixed-age ionization parameters $\log (U)_{0}$ defined before, which then evolves with time according to the $Q(H)$ time evolution (see Rigby \& Rieke 2004). Since $Q(H)$ decreases after the SF quenching, we expect the ionization parameter to get lower as a function of time. For this reason, each model with a given age will be characterized by an evolving-age ionization parameter $\log (U)_{t}$.

For the ionized nebula, we adopt a hydrogen density $n_{\mathrm{H}}=100 \mathrm{~cm}^{-3}$, which is in agreement with the typical densities in observed star-forming regions (Dopita et al. 2000, 2006; Kew01), and the solar chemical composition by Asplund, Grevesse \& Sauval 2005 (the abundances of the various elements adopted in this work are listed in table 1 of Dopita et al. 2006.). In particular, we match the metallicity of the ionized nebula with the metallicity of the ionizing stellar population. ${ }^{1}$ For non-solar metallicities, we linearly rescale the abundance of each element, except for $\mathrm{He}, \mathrm{C}$

\footnotetext{
${ }^{1}$ It is important to note that the solar metallicity of the STARBURST99 (and BC03) models is slightly higher $(Z=0.02)$ than the Asplund et al. (2005) one (i.e. $Z=0.016$ ).
}

and $\mathrm{N}$, for which we assume the metallicity dependences reported in Dopita et al. (2006). Depletion factors are fixed at the same values regardless of metallicity (e.g. Dopita et al. 2006; Nakajima \& Ouchi 2014). This implies that the dust-to-metal ratio is fixed regardless of metallicity, and that the dust-to-gas ratio is proportional to metallicity (e.g. Issa, MacLaren \& Wolfendale 1990; Lisenfeld \& Ferrara 1998; Draine et al. 2007; da Cunha et al. 2010). To account for the presence of dust, we adopt the default ISM grain distribution implemented in CLOUDY. However, throughout this work we always use the intrinsic fluxes provided by the CLOUDY code for all the analysed emission lines, i.e. those that do not require any correction for the dust extinction.

Finally, since the emission lines we are interested in require very high gas kinetic temperature (i.e. $T>20000 \mathrm{~K}$ ) to be produced, we stop the calculation at the point in which the kinetic temperature of the gas has fallen down to $T \sim 4000 \mathrm{~K}$, since at this temperature not even the hydrogen can be ionized.

\section{TESTING THE RELIABILITY OF THE PHOTOIONIZATION MODEL}

Before describing the behaviour of the proposed quenching diagnostics, in this section we verify the reliability of our models comparing them with data and literature.

\subsection{Comparison with data}

We perform the comparison with data using the Baldwin, Phillips \& Terlevich diagram (BPT; Baldwin, Phillips \& Terlevich 1981), which is generally adopted to distinguish star-forming from AGN ionization sources (Veilleux \& Osterbrock 1987; Kew01; Kauffmann et al. 2003b; Stasińska et al. 2006). To verify the consistency of our models with real data, we use a sample of $\sim 174000$ starforming galaxies extracted from the Sloan Digital Sky Survey Data Release 8 (SDSS DR8; e.g. Eisenstein et al. 2011, see Quai et al. , in preparation, for details), classified as star forming on the basis of the BPT diagram itself, using the definition by Kauffmann et al. (2003b). Galaxies in this sample have $0.04 \lesssim z \lesssim 0.21,9 \lesssim$ $\log \left(\mathrm{M} / \mathrm{M}_{\odot}\right) \lesssim 12, \mathrm{~S} / \mathrm{N}$ of the $\mathrm{H} \alpha$ flux $>5$ and of the $\mathrm{H} \beta$ flux $>3$. The threshold of $\mathrm{S} / \mathrm{N}(\mathrm{H} \alpha)$ has been adopted because we expect just quenched galaxy spectra to be still characterized by relatively strong low-ionization lines (see Section 2).

The emission line measurements for each object are derived from the MPA-JHU group (Brinchmann et al. 2004) and are corrected for dust extinction assuming the Calzetti extinction curve (Calzetti et al. 2000) and using the $\mathrm{H} \alpha / \mathrm{H} \beta$ ratio to estimate the nebular colour excess $E(B-V)$. Starting from the selected sample, in the following sections we consider different subsamples of objects, selected according to the $\mathrm{S} / \mathrm{N}$ of the emission lines under analysis. Throughout the work, emission lines characterized by $\mathrm{S} / \mathrm{N}<2$ are regarded as not-detected lines, thus their fluxes are treated as upper limits and set to $2 \sigma$. We consider, in particular, upper limits for objects with $\left[\mathrm{O}_{\text {III }}\right] \lambda 5007$ (hereafter $[\mathrm{O}$ III]) and $[\mathrm{Ne}$ III] undetected. On the contrary, $\mathrm{S} / \mathrm{N}>2$ are associated with emission lines like [O II], [N II] $\lambda 6584$ (hereafter [N II]) and [S II] $\lambda \lambda 6717,6731$ (hereafter [S II]).

As illustrated in Fig. 3, models are able to reproduce the bulk of the data distribution in the BPT star-forming branch, with galaxies spanning the entire range of ionization parameters and metallicities considered in our study. The discrepancy of the uppermost envelope of the SF region could be also due to residual contamination by AGN or composite sources (e.g. Stasińska et al. 2006) or to shortcomings related to models. For instance, recent works have argued that, at 


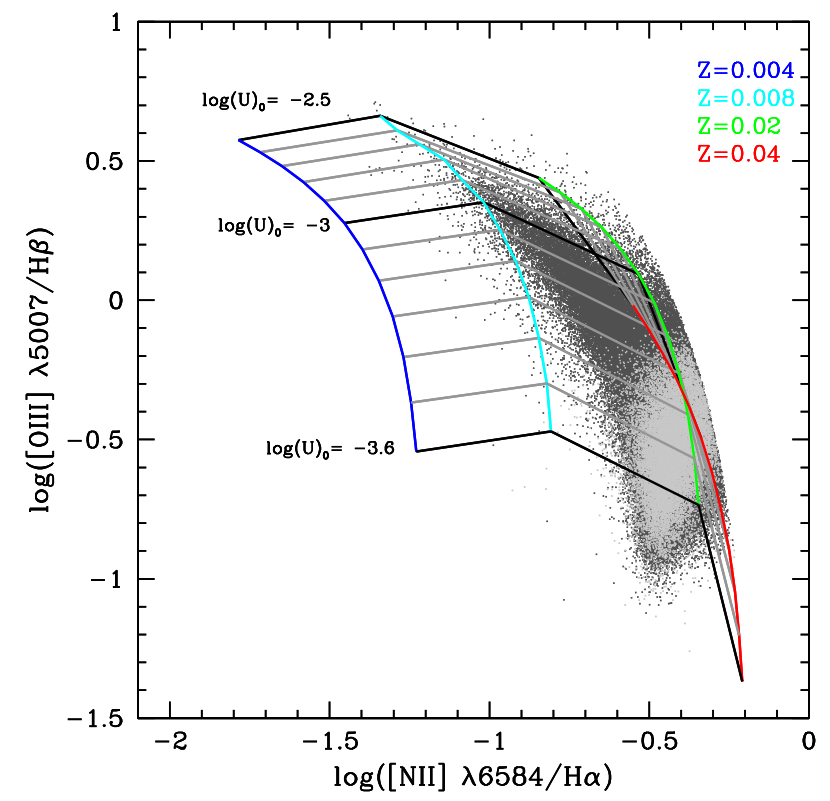

Figure 3. Comparison between our models and observations. Dark grey points are galaxies extracted from the SDSS DR8 with $\mathrm{S} / \mathrm{N}(\mathrm{H} \alpha)>5$, $\mathrm{S} / \mathrm{N}(\mathrm{H} \beta)>3$ and $\mathrm{S} / \mathrm{N}\left(\left[\mathrm{N}_{\mathrm{II}}\right]\right), \mathrm{S} / \mathrm{N}\left(\left[\mathrm{O}_{\mathrm{III}}\right]\right)>2$, while light grey points are galaxies with $\mathrm{S} / \mathrm{N}([\mathrm{O} \mathrm{III}])<2$. The superimposed grid is our set of fixed-age models with different metallicities $(Z=0.004$ blue; $Z=0.008$ cyan; $Z=$ 0.02 green; $Z=0.04$ red) and different $\log (U)_{0}$ (going from -3.6 to -2.5 with steps of 0.1 dex from bottom to top). Black curves mark the levels $\log (U)_{0}=-3.6,-3$ and -2.5 , from bottom to top, as indicated.

the present state, many of the synthetic spectra generally used in the literature can produce too soft UV ionizing fluxes, especially at high metallicities (e.g. Lev10). Moreover, first evidence that local galaxies can have higher N/O ratios and/or higher dust-to-metal ratios especially at high metallicities has been found in the past few years (Brinchmann et al. 2013; Pérez-Montero et al. 2013; Wu et al. 2014; Morisset et al. 2016), suggesting that they could help in filling the gaps between models and data. At $Z>0.02$, models with different metallicities and ionization parameters overlap between each other. This can mainly be attributed to the fact that the $\left[\mathrm{N}_{\text {II }}\right] \lambda 6584 / \mathrm{H} \alpha$ (hereafter $\left[\mathrm{N}_{\text {II }}\right] / \mathrm{H} \alpha$ ) ratio does not have a smooth increase with $\mathrm{Z}$, flattening at supersolar metallicities (see Section 6 for further details). However, defining more sophisticated models able to explain the BPT diagram is beyond the aim of this work and would not influence our studies on the galaxy quenching phases.

It is important to note that the adopted SDSS data are sensitive enough for our methodology, which basically relies on the detection of variations in emission line ratios. Our assumptions of $\mathrm{S} / \mathrm{N}(\mathrm{H} \alpha)>5$ and $\mathrm{S} / \mathrm{N}([\mathrm{O}$ III] $) \leq 2$ allow us to detect differences between these two lines (and thus variations in their ratio)

[O III] and $\mathrm{H} \alpha$ in our sample are $\sim 0.32 \times 10^{-16} \mathrm{erg} \mathrm{s}^{-1} \mathrm{~cm}^{-2}$ and $\sim 1.8 \times 10^{-16} \mathrm{erg} \mathrm{s}^{-1} \mathrm{~cm}^{-2}$, respectively, and the lowest detected $\log ([\mathrm{O} \mathrm{III}] / \mathrm{H} \alpha)$ is $\sim-1.5$, which corresponds to a difference by a factor of $\sim 30$ between the two lines (cf. Quai et al., in preparation). Furthermore, the analysed SDSS individual spectra have a continuum $\mathrm{S} / \mathrm{N}$ around the $\left[\mathrm{O}_{\mathrm{III}}\right]$ line $>5$, which has been proved to be sufficient to provide reasonable measurements of emission line fluxes (e.g. Thomas et al. 2013). This further implies a good sensitivity to variations in ratios involving emission lines in this spectral region.

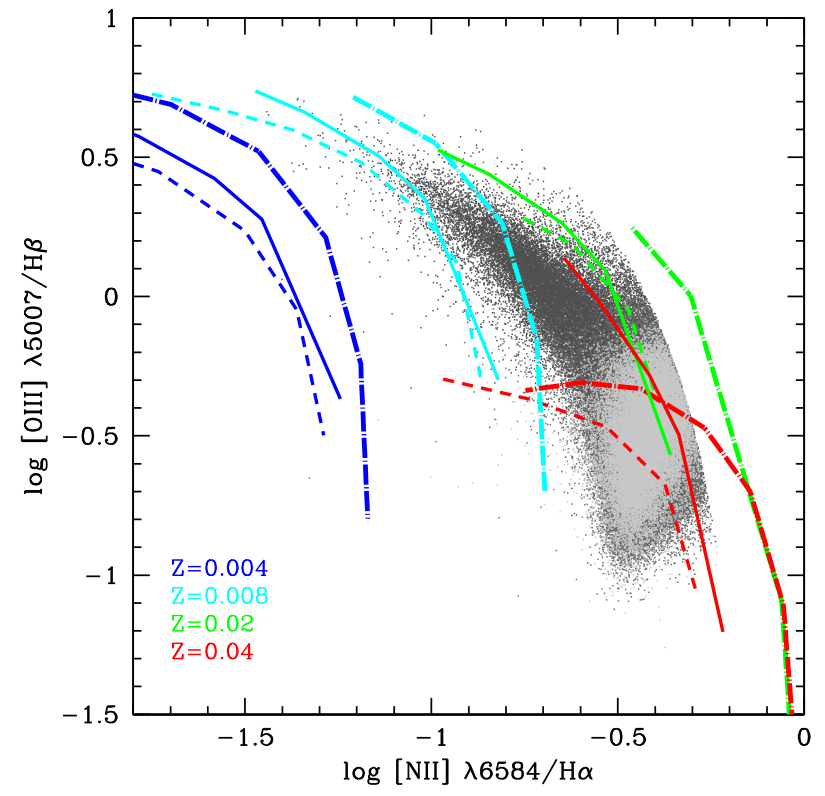

Figure 4. Comparison among our models (solid curves), Lev10 (dashed curves) and Kew01 (dot-dashed curves) predictions, for $-3<\log (U)_{0}<-2$. Grey points are the sample extracted from the SDSS DR8, colour coded as in Fig. 3. Different colours indicate different metallicities $(Z=0.004$, blue; $Z=0.008$ cyan; $Z=0.02$, green; $Z=0.04$, red).

\subsection{Comparison with other models}

We verify the reliability of our models comparing them with other predictions available in the literature: i.e. those presented in Kew01 and those by Lev10. In Table 1 we summarize the main parameters characterizing Kew01, Lev10 and our model.

Fig. 4 shows the results within the BPT plane. Note that, to be consistent with the literature, this comparison is performed only in the case of fixed-age models.

Our models are in reasonable agreement with the literature ones, at each metallicity. However, at $Z \geq 0.02$, the dispersion among different predictions is more pronounced, and in some cases (e.g. Kew01) models produce too high $\left[\mathrm{N}_{\mathrm{II}}\right] / \mathrm{H} \alpha$ values with respect to the data. We also find that, at $Z=0.04$, a difference in the $[\mathrm{O}$ III] $/ \mathrm{H} \beta$ versus $[\mathrm{N}$ II $] / \mathrm{H} \alpha$ slope is present. This can be due to the different dust prescriptions adopted, which lead our models to be dustier than those of Kew01 and Lev10. The discrepancy is larger at higher metallicities, where our models have a higher dust-to-gas ratios with respect to those of the literature (see Section 3). In particular, when many ionizing photons are provided by the central source (i.e. at high $\left.\log (U)_{0} \sim-2\right)$, the larger amount of dust in our models amplifies grain photoelectric heating processes, which produce free electrons able to collisionally excite the [O III]. This increases the $[\mathrm{O} I \mathrm{II}] / \mathrm{H} \beta$ ratio more than the Kev01 and Lev01 models, hence producing a steeper slope for our models. At lower $\log (U)_{0}$, when grain photoelectric heating processes decrease because of the very low number of ionizing photons, there is instead a better agreement among different models.

Note that this does not affect the lowest $\log (U)_{0}$ regimes [i.e. $\left.\log (U)_{0} \lesssim-3.5\right]$, which are the ones relevant in our study. More generally, the discrepancies among different models are related to the different ingredients and photoionization codes adopted for the simulations. In particular, as already mentioned in Section 4.1, different stellar atmospheres can produce UV SEDs with different 
Table 1. Main ingredients of our models, Kew01's model and Lev10's model.

\begin{tabular}{lccc}
\hline & This work & Kew01 & Lev10 \\
\hline Code & CLouDY 13.03 & MAPPINGS III & MAPPINGS III \\
Evolutionary tracks & Geneva High 1994 & Geneva High 1994 & Geneva High 1994 \\
Stellar atmospheres & Lejeune-Shmutz & Lejeune-Shmutz & Paldruach-Hillier \\
$\log (U)_{0}$ & $-3.6<\log (U)_{0}<-2$ & $-3.7<\log (U)_{0}<-2$ & $-3.5<\log (U)_{0}<-1.9$ \\
Ionizing source SFH & SSP & SSP & SSP \\
Ionizing source age & 0.01 Myr & 0.01 Myr & 0.01 Myr \\
Metallicity & $0.001,0.004,0.008,0.02,0.04$ & $0.004,0.008,0.02,0.04$ & $0.004,0.008,0.02,0.04$ \\
\hline
\end{tabular}

slopes, affecting the emission line ratios which are more sensitive to the UV hardness (Schmutz et al. 1992; Hillier \& Miller 1998; Dopita et al. 2000; Pauldrach, Hoffmann \& Lennon 2001; Kew01; Lev10). Furthermore, Kew01 models adopt higher depletion factors for some elements (e.g. $\mathrm{C}$ and $\mathrm{Fe}$ ) and thus higher dust-to-metal ratios for a given metallicity. This produces an increase of the gas electron temperature, which favours the cooling from metal optical lines like [O III] and [N II] (e.g. Shields \& Kennicutt 1995; Charlot \& Longhetti 2001; Brinchmann et al. 2013), increasing their fluxes and thus the emission line ratios in which they are involved.

\section{QUENCHING DIAGNOSTICS}

Before describing how the two emission line ratios mentioned above can help in identifying galaxies which are quenching their SF, in the following sections we describe in more details their behaviour as a function of time, ionization parameter and metallicity, and the relative degeneracies.

\subsection{Emission line ratios and their evolution with time}

The two proposed emission line ratios are expected to suddenly react to the halt of the SF within a star-forming region. In this regard, Figs 5 and 6 show the percentage variation of the emission lines as a function of time from the SF quenching, for different metallicities and initial $\log (U)_{0}$. Regardless of the initial $\log (U)_{0}$, high-ionization lines behave differently from low-ionization ones, with the former changing more significantly as a function of the various parameters. For instance, $[\mathrm{O} \mathrm{III}]$ and $[\mathrm{Ne} \mathrm{III}]$ drop by more than a factor of 10 within the first $2-3 \mathrm{Myr}$ after the SF quenching, especially at the highest metallicity and the lowest $\log (U)_{0}$. Moreover, they experience a temporary rise at $\sim 4 \mathrm{Myr}$, which is particularly pronounced at the highest metallicities. This rise can be attributed to Wolf-Rayet (WR) stars, which are more numerous at higher metallicities (Schaller et al. 1992; see also Lev10) and can supply, on short time intervals, very energetic photons able to re-ionize $\mathrm{O}^{++}$and $\mathrm{Ne}^{++}$, producing a temporary increase of the line fluxes. After 5-6 Myr from the SF quenching, for all metallicities, both $[\mathrm{O}$ III] and [ $\mathrm{Ne}$ III] have declined by a factor of $\sim 100$ with respect to their values in the star-forming phase and, at $\sim 10 \mathrm{Myr}$ they are more than a factor of $\sim 1000$ lower than their initial value.

Compared to [O $\mathrm{OII}]$ and $[\mathrm{Ne} \mathrm{III}], \mathrm{H} \alpha$ and $\left[\mathrm{O}_{\mathrm{II}}\right]$ have a slower decline as a function of time, which is also delayed with respect to high-ionization lines. [O $\mathrm{II}$, which differently from $\mathrm{H} \alpha$ depends on metallicity, is also less dependent on it than [O $\mathrm{III}]$ and $[\mathrm{Ne}$ III]. At $5-6 \mathrm{Myr}$, when the high-ionization lines definitely drop, [O II] and $\mathrm{H} \alpha$ have declined by a factor of $\sim 10$ less, regardless of metallicity. In particular, it takes $\sim 10-15 \mathrm{Myr}$ for the low-ionization lines to decline by a factor of $\sim 100-1000$. These predictions confirm that
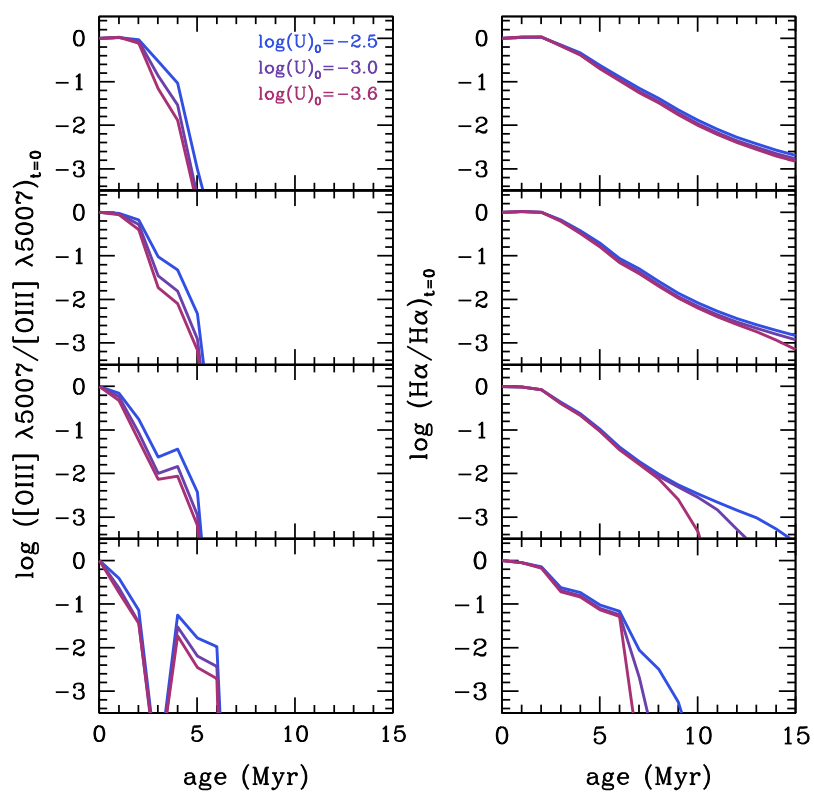

Figure 5. Evolution of the line luminosity relative to the initial one at $t=0$ for [O III] (left) and $\mathrm{H} \alpha$ (right) as a function of time, metallicity and $\log (U)_{0}$. Metallicity $(Z=0.004,0.008,0.02$ and 0.04$)$ increases from the top the bottom panel. In each panel, we show the results for $\log (U)_{0}-2.5,-3$ and -3.6 , with $\log (U)_{0}$ decreasing from blue to red, as indicated.

just quenched galaxies could lack high-ionization emission lines, still having relatively strong low-ionization lines in their spectra.

The behaviour of individual emission lines is reflected in the two emission line ratios considered in our study, as illustrated in Fig. 7. In particular, both $\left[\mathrm{O}_{\mathrm{III}}\right] / \mathrm{H} \alpha$ and $[\mathrm{Ne} \mathrm{III}] /\left[\mathrm{O}_{\mathrm{II}}\right]$ decrease for increasing age and metallicity, at each $\log (U)_{0}$. For both emission line ratios, the decline is more pronounced at higher metallicities, since for a given age, more metallic massive stars have softer UV spectra. Indeed, at $\log (U)_{0}=-3$ and at the lowest $Z$, [O III] $/ \mathrm{H} \alpha$ and $[\mathrm{Ne}$ III $] /\left[\mathrm{O}_{\text {II }}\right]$ decrease by $\sim 0.1$ and $\sim 0.2$ dex within $\sim 2 \mathrm{Myr}$ from the SF quenching, respectively, while at the highest $Z$ they drop by $\sim 1.3$ and $\sim 1$ dex, within the same time interval. However, regardless of metallicity, the two emission line ratios are characterized by a decline by a factor of $\sim 10$ within $\sim 10 \mathrm{Myr}$ from the epoch of the SF quenching. Although fixed-age models assume a fixed shape for the SED of the ionizing source, the ones which are characterized by the lowest $\log (U)_{0}$ can in some sense describe star-forming regions that are quenching their SF, since low-ionization parameters are related to low numbers of ionizing photons and then to low levels of SF. For this reason, it can be interesting to investigate the behaviour of the emission line ratios under analysis as a function of both $\log (U)_{0}$ and $\log (U)_{t}$. Fig. 8 illustrates the case of $[\mathrm{O} I I] / H \alpha$. For $Z=0.02$, a decline by a factor of $\sim 10$ of this ratio corresponds to a decrease in 

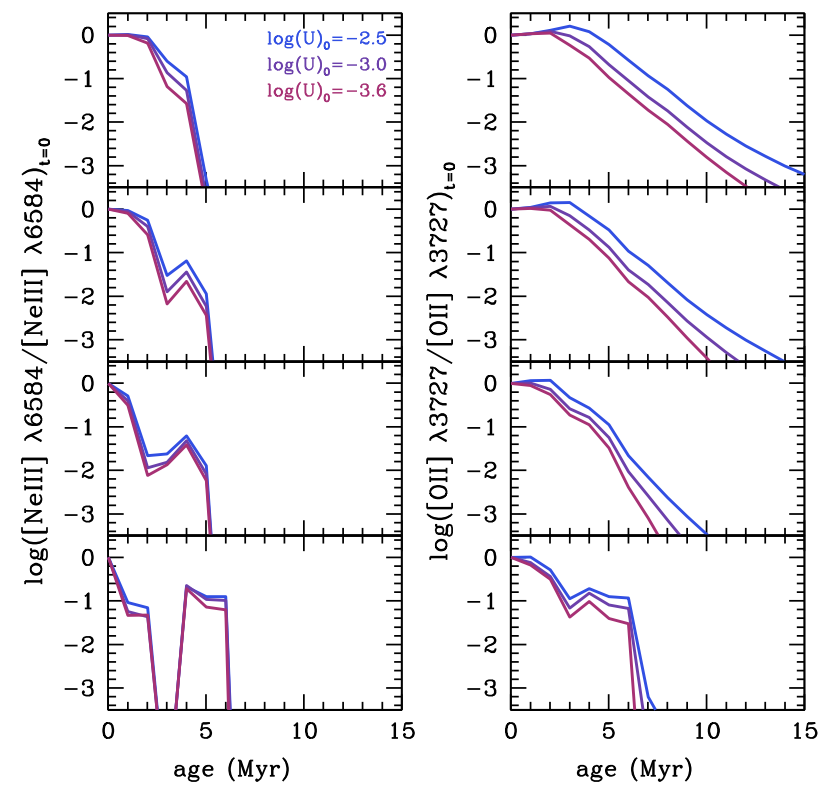

Figure 6. Percentage evolution of [Ne III] (left) and [O II] (right) as a function of time, metallicity and $\log (U)_{0}$. Metallicity $(Z=0.004,0.008,0.02$ and 0.04$)$ increases from the top to the bottom panel. In each panel, we show the results for $\log (U)_{0}-2.5,-3$ and -3.6 , with $\log (U)_{0}$ decreasing from blue to red, as indicated.
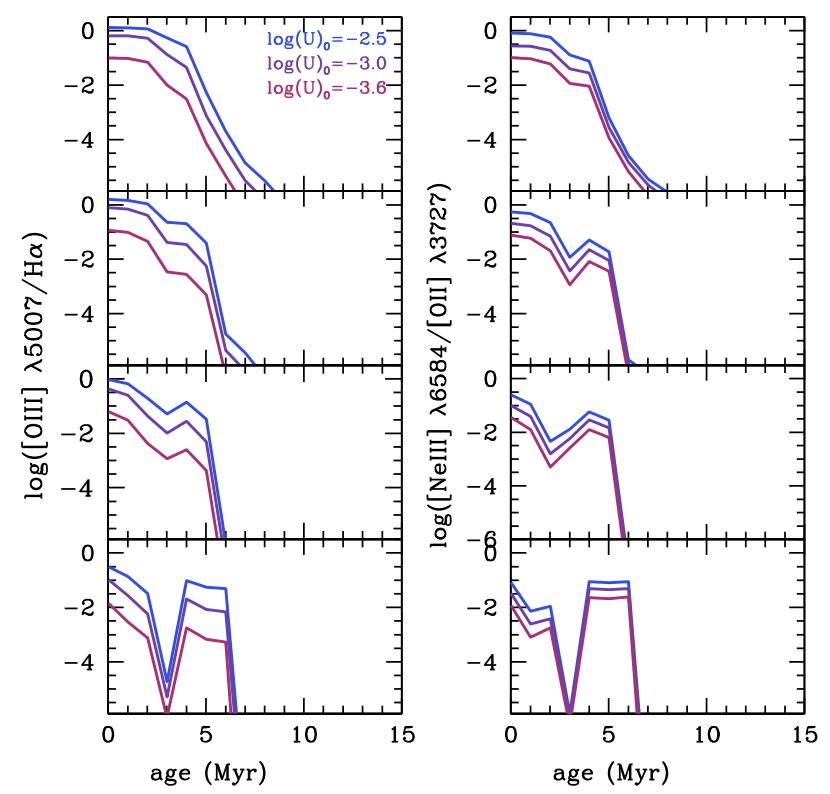

Figure 7. $[\mathrm{O} I I] / \mathrm{H} \alpha$ (left) and $[\mathrm{Ne} \mathrm{III}] /[\mathrm{O} \Pi]$ (right) evolution as a function of time, metallicity and $\log (U)_{0}$. Metallicity $(Z=0.004,0.008,0.02$ and $0.04)$ increases from the top to the bottom panel. In each panel, we show the results for $\log (U)_{0}-2.5,-3$ and -3.6 , with $\log (U)_{0}$ decreasing from blue to red, as indicated.

$\log (U)_{t}$ by 0.1 dex [starting from an initial $\log (U)_{0}=-3$ ], within a time interval of $\sim 2 \mathrm{Myr}$, while only a more pronounced decrease by $\sim 1$ dex in $\log (U)_{0}$ can produce the same effect. Therefore, the decline of $[\mathrm{O} \mathrm{III}] / \mathrm{H} \alpha$ is more rapid for evolving-age models than for fixed-age ones. This is due to the fact that the former include the additional effect of the UV softening as a function of time.

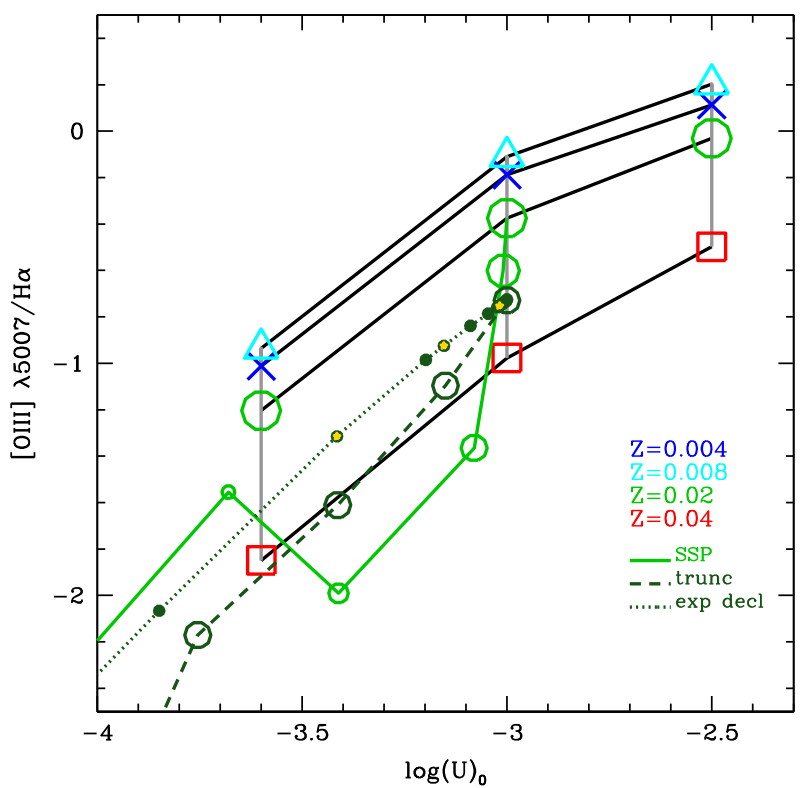

Figure 8. $[\mathrm{O} \mathrm{III}] / \mathrm{H} \alpha$ as a function of $\log (U)_{0}$ and $\log (U)_{t}$. Grey curves connect models with the same $\log (U)_{0}$, for the three $\log (U)_{0}=-3.6$, -3 and -2.5 and different metallicity $(Z=0.004$ blue; $Z=0.008$ cyan; $Z=$ 0.02 green; $Z=0.04$ red), while black curves connect models with the same metallicity. For $Z=0.02$, evolving-age models for SSP (light green empty circles), truncated (dark green empty circles) and the exponentially declining (dark green filled circles) SFHs are shown (see Section 5.4 for further details), for an initial $\log (U)_{0}=-3$. The emission line ratio evolution is illustrated with a time-step of $1 \mathrm{Myr}$ within the first $10 \mathrm{Myr}$ after quenching, $\sim 20$ Myr from 10 to $100 \mathrm{Myr}$ after quenching, and $100 \mathrm{Myr}$ even further. For the exponentially declining SFH, small gold stars mark the values of the emission line ratios corresponding to 10,80 and $200 \mathrm{Myr}$ after the SF quenching, from the highest to the lowest value of $[\mathrm{O}$ III $] / \mathrm{H} \alpha$.

\subsection{The influence of different hydrogen densities}

The typical hydrogen densities of $\mathrm{H}_{\mathrm{HI}}$ regions are of the order of $n_{\mathrm{H}} \sim 100 \mathrm{~cm}^{-3}$, and are therefore well below the critical densities of the forbidden lines considered in our analysis. ${ }^{2}$ However, it can be interesting to investigate how $\left[\mathrm{O}_{\mathrm{III}}\right] / \mathrm{H} \alpha$ and $[\mathrm{Ne} \mathrm{III}] /\left[\mathrm{O}_{\mathrm{II}}\right]$ are affected by an increase of $\mathrm{n}_{\mathrm{H}}$ towards the critical densities $\left(n_{\text {crit }}\right)$ of [O III], [Ne III] and [O II]

As illustrated in Fig. 9, we find that $[\mathrm{O} \mathrm{III}] / \mathrm{H} \alpha$ increases by only $\sim 0.2$ dex for $n_{\mathrm{H}}<n_{\text {crit }}([\mathrm{O}$ III] $]$, i.e. when $\mathrm{H} \alpha$ and [O III] are both increasing due to the increment of ionized hydrogen and free electrons, which are able to excite metal ions. On the contrary, when $n_{\mathrm{H}}>n_{\text {crit }}\left(\left[\mathrm{O}_{\mathrm{III}}\right]\right),[\mathrm{O} \mathrm{III}] / \mathrm{H} \alpha$ rapidly drops due to the prevalence of collisional de-excitations for the [O $\mathrm{III}]$ line.

The $[\mathrm{Ne} \mathrm{III}] /[\mathrm{O}$ II] ratio has a more complex behaviour, since it involves two forbidden lines. In particular, it increases by $\sim 0.5$ dex for $n_{\mathrm{H}}<n_{\text {crit }}([\mathrm{O}$ II] $)$, while it starts to rapidly increase when $n_{\text {crit }}([\mathrm{O} I \mathrm{II}])$ is reached and [O $\left.\mathrm{II}\right]$ saturates. This trend is also inverted when $n_{\text {crit }}([\mathrm{Ne}$ III $])$ is reached.

In general, we find that zero-age models with high- $\mathrm{n}_{\mathrm{H}}$ can produce the same $[\mathrm{O} I I I] / \mathrm{H} \alpha$ values as quenching ones with low$\mathrm{n}_{\mathrm{H}}$ (and zero age models with low- $\mathrm{n}_{\mathrm{H}}$ can produce the same $[\mathrm{Ne} \mathrm{III}] /[\mathrm{O}$ II $]$ values as quenching ones with high- $\mathrm{n}_{\mathrm{H}}$ ), at each metallicity. This means that our models can in principle be affected by ${ }^{2} n_{\text {crit }[\mathrm{OIII}]} \sim 7 \times 10^{5} \mathrm{~cm}^{-3}, n_{\text {crit [Ne III] }]} \sim 1.1 \times 10^{7} \mathrm{~cm}^{-3}, n_{\text {crit [O II] }} \sim 1 \times$
$10^{4} \mathrm{~cm}^{-3}$. 

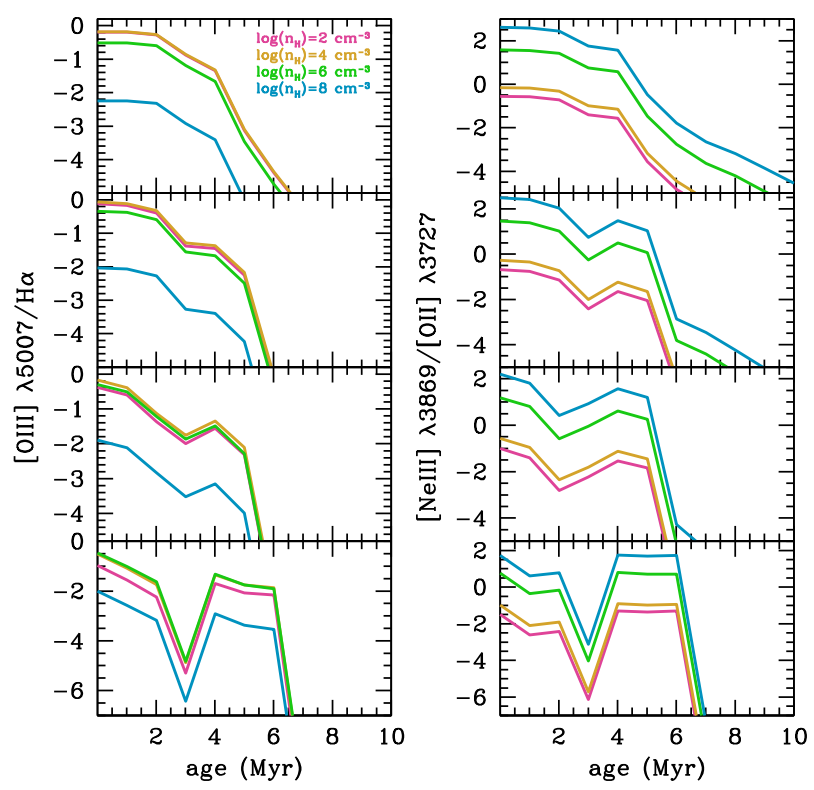

Figure 9. $[\mathrm{O} \mathrm{III}] / \mathrm{H} \alpha$ and $[\mathrm{Ne} I I] /\left[\mathrm{O}_{\mathrm{II}}\right]$ time evolution for different values of $\mathrm{n}_{\mathrm{H}}$, and $\log (U)_{0}=-3$. The hydrogen density increases from $\log \left(\mathrm{n}_{\mathrm{H}}\right)=2$ (which is our default value) to $\log \left(\mathrm{n}_{\mathrm{H}}\right)=8$, from pink to cyan, as labelled. Metallicity $(Z=0.004,0.008,0.02$ and 0.04$)$ increases from the top to the bottom panel.

the $n_{H}$ degeneracy. However, this holds true for $n_{H} \gtrsim 4$, which is much larger than the typical $\mathrm{n}_{\mathrm{H}}$ in $\mathrm{H}$ II regions. This allows us to exclude the very low $[\mathrm{O}$ III] $/ \mathrm{H} \alpha$ and $[\mathrm{Ne}$ III $] /[\mathrm{O}$ II] found in this work to be related to density rather than quenching effects. Finally, we notice that the sudden drop of the emission line ratios after a few Myr from the SF quenching is present regardless of $\mathrm{n}_{\mathrm{H}}$.

\subsection{The influence of different synthetic stellar spectra}

In this section we verify if the results illustrated in Section 5.1 are influenced by the use of different synthetic spectra to describe the central ionizing source. Fig. 10 illustrates a comparison between STARBURST 99 and BC03 models, in the case of $\log (U)_{0}=-3$ and for the four metallicities considered in this work. The general behaviour of $\left[\mathrm{O}{ }_{\mathrm{III}}\right] / \mathrm{H} \alpha$ and $[\mathrm{Ne} \mathrm{III}] /\left[\mathrm{O}_{\mathrm{II}}\right]$ obtained using $\mathrm{BC0} 3$ models is in agreement with the ones resulting from STARBURST 99 spectra. Both emission line ratios decline by a factor of $\sim 10$ within $\sim 10 \mathrm{Myr}$ from the quenching of the SF, at each metallicity. After the SF shutdown, the behaviour of the two emission line ratios is more uneven in the case of BC03 models than for STARBURST 99 ones. This is probably due to the different stellar atmospheres (see Charlot \& Longhetti 2001) and evolutionary tracks (see Bruzual \& Charlot 2003) used to compute the models. For instance, BC03 models are based on the Padova1994 evolutionary tracks (Bressan, Chiosi \& Fagotto 1994), which are characterized by a higher number of WR stars with respect to the Geneva-HIGH 1994 ones (as discussed in Bruzual \& Charlot 2003). This can give more pronounced discontinuities in the emission line ratios at the very early ages after the SF quenching. The time at which the two emission line ratios begin to definitely drop is the same in the case of BC03 and STARBURST 99 spectra. After this epoch, the time required to have a decrease by a factor of $\sim 1000$ is slightly higher for BC03 models, with a shift of $\sim 1-3 \mathrm{Myr}$ for both emission line ratios. Apart from these small differences, we can conclude that our methodology is valid also when BC03 models
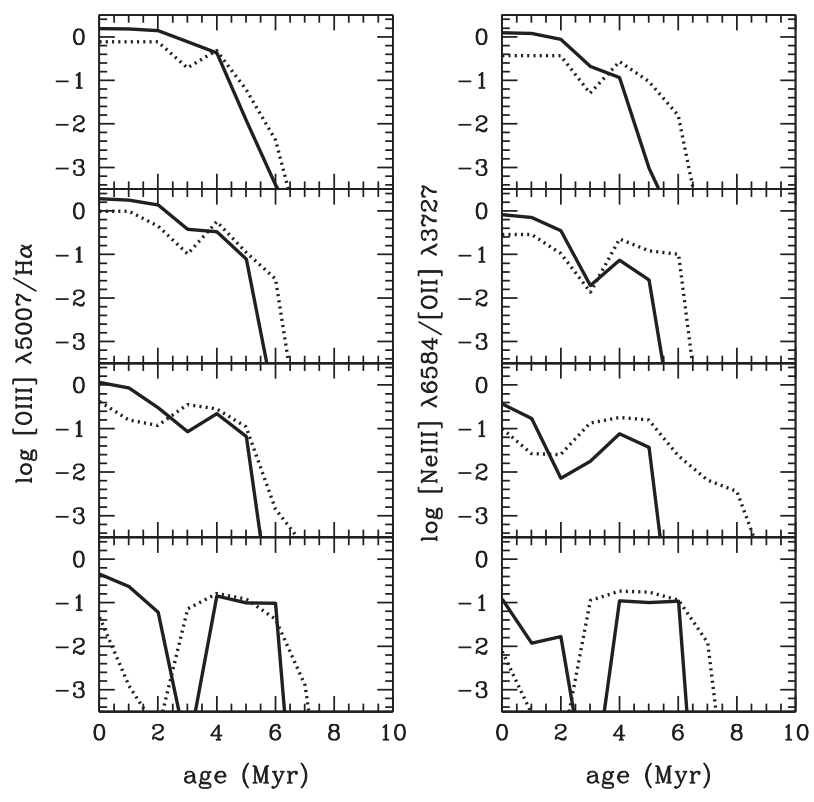

Figure 10. Comparison between $\left[\mathrm{O}_{\mathrm{III}}\right] / \mathrm{H} \alpha$ and $[\mathrm{Ne} \mathrm{III}] /\left[\mathrm{O}_{\mathrm{II}}\right]$ obtained assuming STARBURST99 (solid curves) and BC03 (dotted curves) models to simulate the central ionizing source, assuming $\log (U)_{0}=-3$. Metallicity $(Z=0.004,0.008,0.02,0.04)$ increases from the top to the bottom panel.

are adopted. This is an interesting result, since BC03 models are generally used only to describe more advanced evolutionary phases of galaxy evolution (e.g. Leitherer et al. 1999; Bruzual \& Charlot 2003; Chen et al. 2010).

\subsection{The influence of different star formation histories}

The instantaneous SF quenching modelled so far can be considered an extreme case, which allows us to better show the strength of our approach to probe SF quenching on very short time-scales. In this section, we investigate how the behaviour of $[\mathrm{O} \mathrm{III}] / \mathrm{H} \alpha$ and $[\mathrm{Ne} \mathrm{III}] /\left[\mathrm{O}_{\mathrm{II}}\right]$ changes if different star-formation histories (SFHs) and more realistic and smoother SFHs are assumed. In particular, using STARBURST99 models, we analyse the case of a truncated SFH, with SFR $=1 \mathrm{M}_{\odot} \mathrm{yr}^{-1}$ up to $200 \mathrm{Myr}$ and zero at older ages, and the case of a smoother decline described by an exponentially declining SFR (i.e. SFR $\propto \mathrm{e}^{-t / \tau}$ ), with $\tau=200 \mathrm{Myr}$, since this is the SFH shape generally assumed to describe local star-forming galaxies (e.g. Bell $\&$ de Jong 2001). For the truncated SFH, an SFR $=1 \mathrm{M}_{\odot} \mathrm{yr}^{-1}$ is chosen to match the typical SFRs of SDSS star-forming galaxies (Brinchmann et al. 2004; Whitaker et al. 2014) at stellar masses comparable with the ones of our sample. For the exponentially declining SFH, models are normalized to $10^{6} \mathrm{M}_{\odot}$. Moreover, we do not consider very high values of $\tau>200 \mathrm{Myr}$, since in this case the SFH would extend at much larger times, incompatible with our assumption that galaxies are quenching their SF. Furthermore, larger $\tau$ s have been demonstrated to produce galaxies which never leave the blue cloud (e.g. Schawinski et al. 2014).

For both SFHs, we consider $Z=0.02$ and an initial ionization level $\log (U)_{0}=-3$. In particular, to be consistent with the SSP case (see Section 5.1), for the truncated SFH we attribute $\log (U)_{0}=-3$ at the time corresponding to the last act of SF (i.e. $200 \mathrm{Myr}$ in this case). For the exponentially declining $\mathrm{SFH}, \log (U)_{0}=-3$ is instead associated with the age at which $Q(H)$ begins to drop ( $\sim 10 \mathrm{Myr}$ ), since we are interested in the epochs at which the SF starts to quench. Moreover, these choices allow us to avoid, at very 


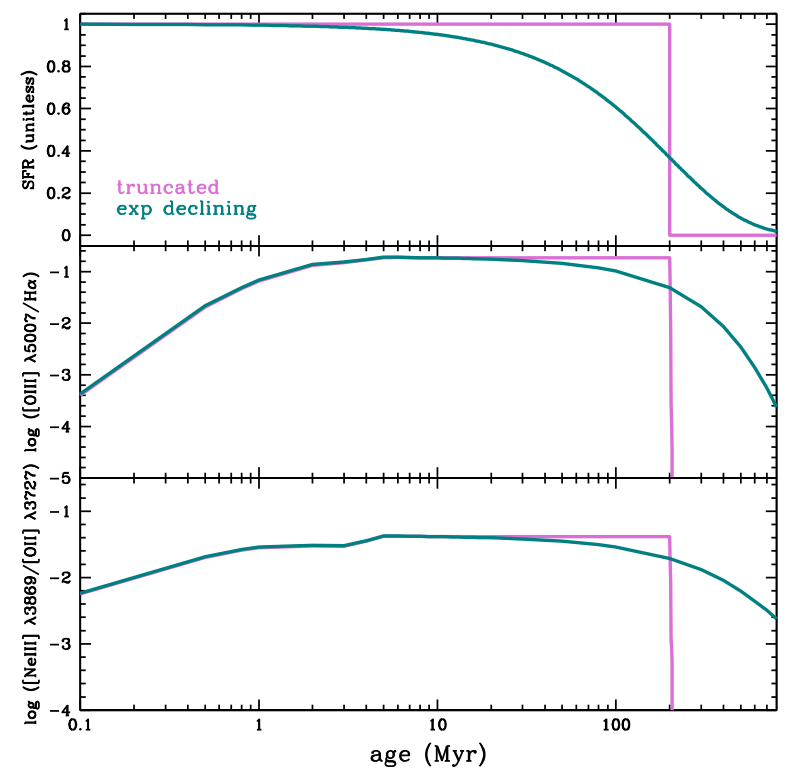

Figure 11. SFR, $\left[\mathrm{O}_{\mathrm{III}}\right] / \mathrm{H} \alpha$ and $\left[\mathrm{Ne} \mathrm{III}^{\mathrm{II}}\right] /\left[\mathrm{O}_{\mathrm{II}}\right]$ emission line ratios as a function of time for the truncated and the exponentially declining SFHs, $Z=$ 0.02 and $t=0$. In the top panels, the SFRs have different scales due to the different definitions of the two SFHs (described in the text). In both cases of truncated and exponentially declining $\mathrm{SFH}$, the fast rise before the time of quenching is visible, as described in the text.

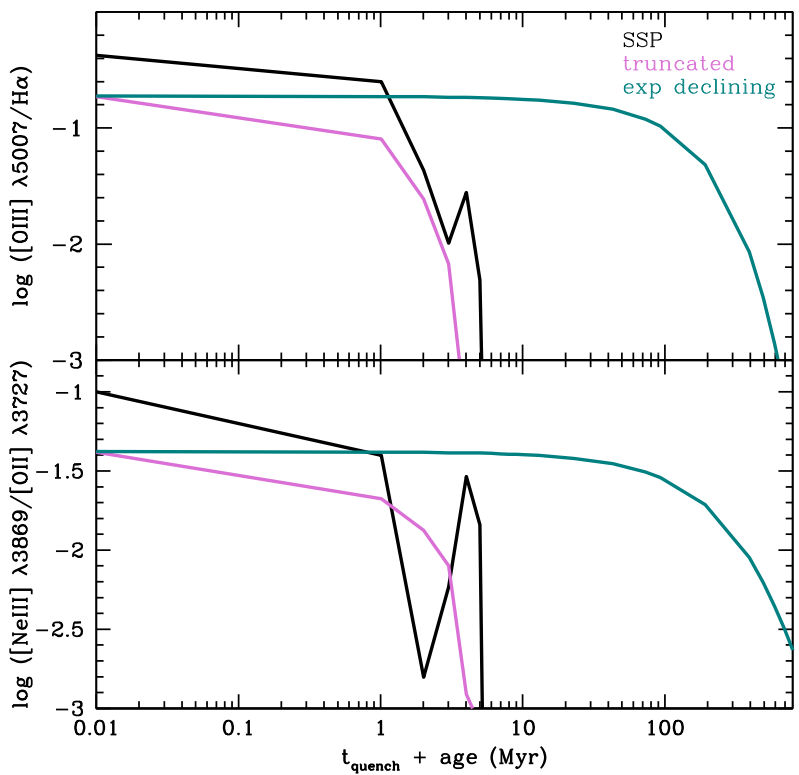

Figure 12. $\left[\mathrm{O}_{\mathrm{III}}\right] / \mathrm{H} \alpha$ and $[\mathrm{Ne} \mathrm{III}] /\left[\mathrm{O}_{\mathrm{II}}\right]$ emission line ratios as a function of time for different SFHs and $Z=0.02$. The SSP (black curve), truncated (violet curve) and the exponentially declining (green curve) SFHs are shown up to $\sim 800 \mathrm{Myr}$ from the time of quenching (indicated as $t_{\text {quench }}$ ).

early times $t<t_{\mathrm{MS}}\left(\mathrm{O}\right.$ stars) (i.e. $\sim 2 \times 10^{7} \mathrm{yr}$ ), the short transient phase during which a rapid rise of ionizing photons characterizes both SFHs (see Madau, Pozzetti \& Dickinson 1998), also reflecting on the two emission line ratios under analysis (see Fig. 11).

Fig. 12 shows the time evolution of $\left[\mathrm{O}\right.$ III] $/ \mathrm{H} \alpha$ and $\left[\mathrm{Ne}\right.$ III] $/\left[\mathrm{O}_{\mathrm{II}}\right]$ for the three assumed SFHs, starting from the time of quenching. For the truncated SFH stopping at $200 \mathrm{Myr}$, the two emission line ratios drop by a factor of $\sim 10$ within $\sim 2 \mathrm{Myr}$ and by more than a factor of $\sim 1000$ within $\sim 10 \mathrm{Myr}$ from the quenching of the SF, similarly to the SSP case.

In the case of an exponentially declining SFR, instead, they decrease following the decline of the SFR. In particular, we find that the star-forming region takes $\sim 80 \mathrm{Myr}$ to become quiescent, reaching specific star-formation rate (sSFR) $\sim 10^{-11} \mathrm{yr}^{-1}$ (which are typical of quiescent galaxies) and, within this time interval, both $[\mathrm{O}$ III $] / \mathrm{H} \alpha$ and $[\mathrm{Ne}$ III $] /[\mathrm{O}$ II $]$ decrease by a factor of $\sim 2$. This decline corresponds to a decrease in $\log (U)_{t}$ by only $\sim 0.2 \mathrm{dex}$, as illustrated in Fig. 8, implying that, when smoother SFHs are considered, $\log (U)_{t}$ has a smoother decline. Moreover, $\sim 500$ Myr are necessary for the two emission line ratios to decline by a factor of $\sim 10$.

It is interesting to note that the value of the two emission line ratios at the age at which the SF stops (200 Myr for the truncated and $\sim 10$ Myr for the exponentially declining SFH) is lower by a factor of $\sim 2$ for more complex SFHs than for a 0.01 Myr SSP with the same $\log (U)_{0}=-3$. Therefore, if an ionizing stellar population forms stars continuously on a longer time interval, its SED at the time of quenching is softer than the SED of a stellar population which forms all its stars into a single burst. This can be due to the accumulation of long lived stars contributing mostly to the flux at longer wavelengths. Moreover, the temporary increase of $[\mathrm{O}$ III] $] / \mathrm{H} \alpha$ and $[\mathrm{Ne} \mathrm{III}] /[\mathrm{O}$ II] due to WR stars is less evident for more complex SFHs than for the SSP case, since, even after the SF quenching, the evolution is dominated by the numerous generations of stars formed during time rather than by the short-lived WR stars.

All these trends confirm that the decline of $[\mathrm{O} \mathrm{III}] / \mathrm{H} \alpha$ and $[\mathrm{Ne}$ III $] /[\mathrm{O}$ II $]$ takes place regardless of the shape of the assumed SFH and that very low values of these emission line ratios are expected whether galaxies have abruptly quenched their SF or they have gradually reached low levels of SF.

\subsection{The time evolution of optical colours}

The softening of the SED associated with the SF quenching also implies an evolution of galaxy colours with time. Since we are analysing optical SDSS spectra, in this section we focus on the intrinsic $(u-r)$ optical colour, showing its evolution as a function of time in Fig. 13. In particular, the three analysed quenching SFHs are compared to a continuous SFH with $\mathrm{SFR}=1 \mathrm{M}_{\odot} \mathrm{yr}^{-1}$ and to a quenching SFH with $\mathrm{SFR}=1 \mathrm{M}_{\odot} \mathrm{yr}^{-1}$ until $500 \mathrm{Myr}$ and zero at later ages. The colour reddening is fast for the quenching SFHs, and colours are always redder than the colours of a continuous SF model even when a significant population of old stars has already been built up (i.e. after $\sim 500 \mathrm{Myr}-1 \mathrm{Gyr})$. We find that the typical $(u-r)$ colours of green valley [1.5 $\lesssim(u-r) \lesssim 2.5$, depending on mass] or red sequence $[(u-r) \gtrsim 2-3]$ galaxies (Schawinski et al. 2014) are reached at later times (i.e. $\sim 1 \mathrm{Gyr}$ ) with respect to the timescales considered so far (i.e. $\sim 10-100 \mathrm{Myr}$ ). This happens even when an old stellar population is already present for a continuous SF model at the time of the SF halt (as in the case of the $500 \mathrm{Myr}-$ truncated SFH). It is important to note that all these trends refer to an individual quenching stellar population and could thus be diluted by the mutual effects of the multiple stellar populations inside a galaxy which are experiencing different evolutionary stages.

\subsection{The expected fractions of quenching candidates}

In the previous sections we found that $[\mathrm{O}$ III $] / \mathrm{H} \alpha$ and $[\mathrm{Ne}$ III] $] /[\mathrm{O} I \mathrm{II}]$ are very sensitive to the SF quenching and that star-forming galaxies characterized by low values of these two emission line ratios might be objects caught in the act of quenching. However, the precise 

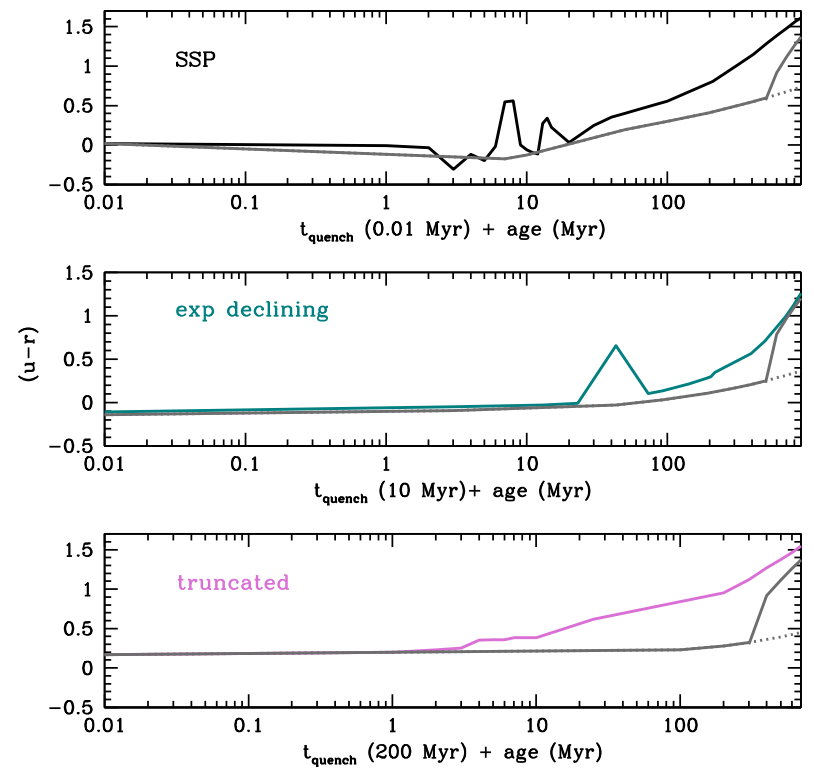

Figure 13. Time evolution of the optical $(u-r)$ colour, for solar metallicity and different SFHs. Black, violet and green curves refer to the SSP, truncated and exponentially declining SFHs considered in this work. Grey dotted and solid curves refer to a continuous $\mathrm{SFH}$ with $\mathrm{SFR}=1 \mathrm{M}_{\odot} \mathrm{yr}^{-1}$ and to a truncated SFR with SFR $=1 \mathrm{M}_{\odot} \mathrm{yr}^{-1}$ until $500 \mathrm{Myr}$ and zero at later ages, respectively. From top to bottom, we show the colour evolution within $900 \mathrm{Myr}$ from the quenching time of the SSP (i.e. $0.01 \mathrm{Myr}$ ), exponential ( $\sim 10 \mathrm{Myr})$ and truncated $(\sim 200 \mathrm{Myr})$ SFHs considered in this work.

time-scales that the emission line ratios are able to trace depend on the SFH of the central ionizing source. Deriving these timescales can be useful to understand what is the physical phenomenon associated with the SF quenching and to estimate the fraction of galaxies observed in the phase of quenching. In particular, the faster the $\mathrm{SF}$ is quenched, the faster the galaxies move from the blue cloud to the red sequence, and the less they clump in the transition zone. To estimate the percentages of objects which are expected to be in the quenching phase, we use the following equation:

$F_{\mathrm{QG}}=t_{Q} / t_{\mathrm{DM}}=t_{Q} \times \mathrm{sSFR}$,

where $F_{\mathrm{QG}}$ is the expected fraction of quenching galaxies, $t_{Q}$ is the time needed for the emission line ratios to decrease by a factor of $\sim 10$ (i.e. $\sim 10 \mathrm{Myr}$ for the SSP/truncated case and $\sim 500 \mathrm{Myr}$ for the exponentially declining SFH; see Section 5.1) and $t_{\mathrm{DM}}$ is the doubling-mass time (e.g. Guzmán et al. 1997; Madau \& Dickinson 2014), which we consider as a proxy of the typical lifetime of an SF galaxy (Guzmán et al. 1997; Greis et al. 2016). In particular, since the typical sSFRs of SF galaxies with redshift and masses comparable to ours [i.e. $0.2<z<0.4$ and $9<\log \left(\mathrm{M} / \mathrm{M}_{\odot}\right)<12$ ] range from $\sim 10^{0.3} \mathrm{Gyr}^{-1}$ to $\sim 10^{0.1} \mathrm{Gyr}^{-1}$ (e.g. Karim et al. 2011), we can deduce that the typical $t_{\mathrm{DM}}$ for our sample ranges from $\sim 3$ to $\sim 10 \mathrm{Gyr}$, increasing for increasing mass.

The expected fractions of quenching galaxies are $\sim 0.06$ 0.2 per cent for the SSP/truncated SFHs and $\sim 5-15$ per cent for the exponentially declining one, respectively, decreasing for increasing mass. It is interesting to note that, once that a complete sample of quenching candidates is selected from an SF galaxy sample, the observed fractions $F_{\mathrm{QG}}$ derived from data can help in disentangling what are the effective time-scales of the quenching mechanism at work. However, degeneracies (see Section 6) and especially observational limits (e.g. the limiting fluxes of the analysed emission line) can hamper the detections of the whole population of existing

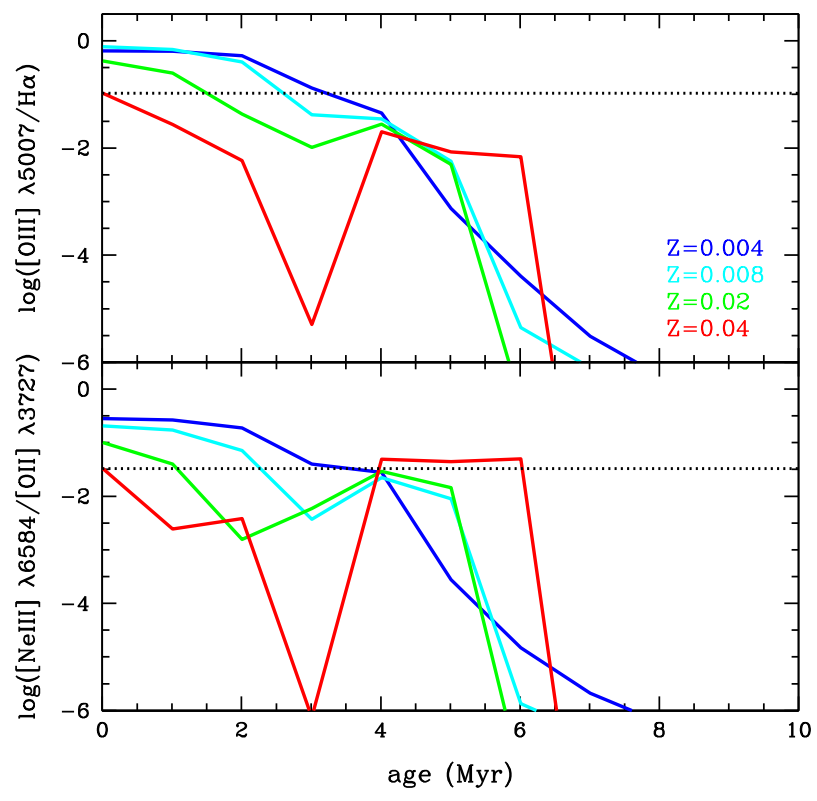

Figure 14. $[\mathrm{O} \mathrm{III}] / \mathrm{H} \alpha$ (top) and $[\mathrm{Ne}$ III] $] /[\mathrm{O}$ II] (bottom) as a function of time, for $\log (U)_{0}=-3$, and different metallicities $(Z=0.004$, blue; $Z=0.008$, cyan; $Z=0.02$, green; $Z=0.04$, red). In each panel, the black dotted line marks the initial value of the emission line ratios for $Z=0.04$.

quenching candidates. Therefore, the derived percentages should be considered as lower limits, as well as the inferred quenching time-scales.

\section{MITIGATING THE IONIZATION-METALLICITY DEGENERACY}

One of the limitations of our methodology is the metallicity degeneracy. Indeed, since higher metallicity ionizing SEDs are softer than lower metallicity ones due to the larger opacity of the ionizing stars (see Fig. 2), they can have the same effects on the emitted spectrum and emission line ratios as quenched, low-ionization ones. Reducing this degeneracy is extremely important for our analysis, in order to exclude objects whose $[\mathrm{O}$ III $] / \mathrm{H} \alpha$ and $[\mathrm{Ne}$ III] $/[\mathrm{O}$ II] are low because of high metallicities rather than low ionization. The main effect of the metallicity degeneracy is illustrated in Fig. 14, for $\log (U)_{0}=-3$. The same value of an emission line ratio can be produced by different combinations of age [i.e. $\left.\log (U)_{t}\right]$ and metallicity. In particular, younger star-forming regions with higher metallicity can produce the same results as older star-forming regions with lower metallicity. This occurs for both $[\mathrm{O}$ III] $] / \mathrm{H} \alpha$ and $[\mathrm{Ne}$ III]/[O II] .

To mitigate the effects of the metallicity degeneracy, we suggest the use of independent pairs of emission line ratios, depending separately on ionization and metallicity as much as possible. In particular, we combine $[\mathrm{O} I I I] / \mathrm{H} \alpha$ and $[\mathrm{Ne} I I I] /[\mathrm{O} I I]$ with the [N $\mathrm{NI}_{\text {II }} \lambda 6584 /\left[\mathrm{O}_{\text {II }}\right] \lambda 3727$ (hereafter $\left[\mathrm{N}_{\text {II }}\right] /\left[\mathrm{O}_{\text {II }}\right]$ ) emission line ratio, whose behaviour in our model as a function of the stellar metallicity is illustrated in Fig. 15, for a fixed age of $0.01 \mathrm{Myr}$. Here we also compare $\left[\mathrm{N}_{\mathrm{II}}\right] /\left[\mathrm{O}_{\mathrm{II}}\right]$ with other two metallicity indicators, i.e. $\left[\mathrm{N}_{\Pi}\right] / \mathrm{H} \alpha$ (e.g. Denicoló, Terlevich \& Terlevich 2002; Pettini \& Pagel 2004; Nagao, Maiolino \& Marconi 2006) and [N II] $\lambda 6584 /[\mathrm{S}$ II] $\lambda \lambda 6717,6731$ (e.g. Nagao et al. 2006) (hereafter $\left[\mathrm{N}\right.$ II]/[S II]). While the $\left[\mathrm{N}\right.$ II] $/ \mathrm{H} \alpha$ and $\left[\mathrm{N}_{\text {II }}\right] /\left[\mathrm{S}_{\mathrm{II}}\right]$ ratios flatten at high metallicities (Kewley \& Dopita 2002; Lev10) and have larger dependences on the ionization level, $[\mathrm{N}$ II] $]\left[\mathrm{O}_{\mathrm{II}}\right]$ scales smoothly from low 


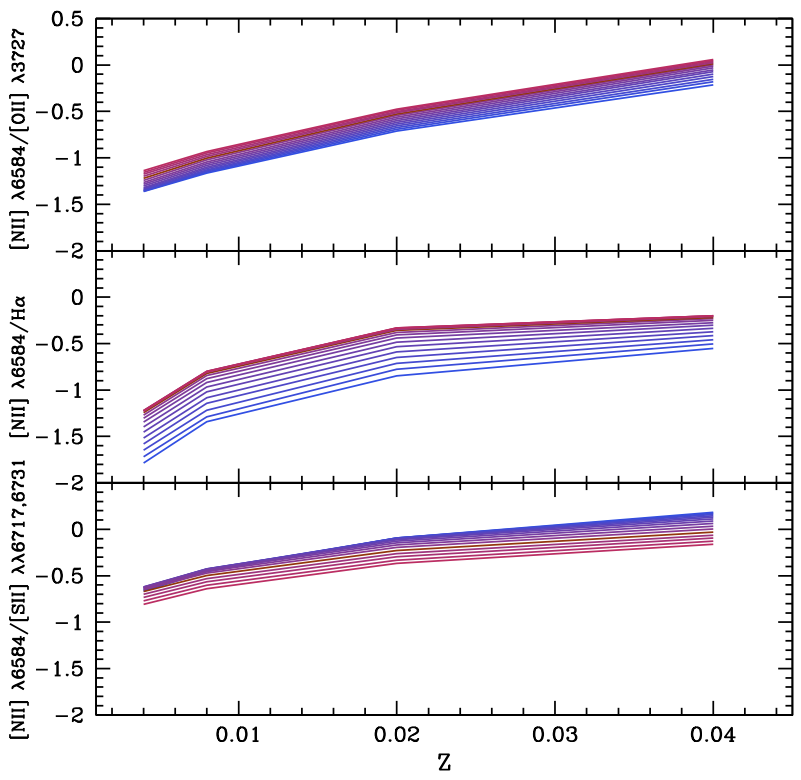

Figure 15. Comparison among $[\mathrm{N} I I] /\left[\mathrm{O}_{I I}\right],[\mathrm{N} I I] / \mathrm{H} \alpha$ and $[\mathrm{N} I I] /\left[\mathrm{S}_{I I}\right]$ as a function of metallicity and $\log (U)_{0}$. Colours are coded as in Fig. 5 .

to high abundances and has a little dependence on $\log (U)_{0}$ (e.g. van Zee et al. 1998; Dopita et al. 2000; Kewley \& Dopita 2002). Moreover, even if the $\left[\mathrm{N}_{\mathrm{II}}\right] /\left[\mathrm{S}_{\mathrm{II}}\right]$ ratio is less affected by reddening effects (it involves emission lines which are closer in wavelength), it has a weaker dependence on metallicity with respect to [N $\mathrm{II}] /[\mathrm{O}$ II], which has also the advantage of including stronger, and thus more easily detectable, emission lines. Therefore, it can be preferable once that the observed emission lines are corrected for dust extinction. In the following, in order to mitigate the metallicity degeneracy, we combine the two proposed quenching diagnostics with the $[\mathrm{N} \mathrm{II]/[O} \mathrm{II]}$ ratio.

\section{IDENTIFYING GALAXIES IN THE QUENCHING PHASE}

In the previous sections we found that galaxies which have quenched their $\mathrm{SF}$ in the recent past can be characterized by low values of the $\left[\mathrm{O}_{\mathrm{III}}\right] / \mathrm{H} \alpha$ and the $[\mathrm{Ne} \mathrm{III}] /\left[\mathrm{O}_{\mathrm{II}}\right]$ emission line ratios. In this section, we test the reliability of our approach by applying it to the SDSS sample described in Section 4.1, and analysing the properties of some of the extreme candidates selected. However, it is important to remind that it is beyond the scope of this work to identify a complete sample of quenching candidates and to investigate their detailed properties, for which we refer the interested readers to a companion paper (Quai et al., in preparation).

Our methodology consists in selecting galaxies with high $\mathrm{S} / \mathrm{N}$ $\mathrm{H} \alpha$ in their spectra and lying at the lowest envelope [i.e. lowest $\left.\log (U)_{0}\right]$ of the SF galaxy distribution within the $[\mathrm{O}$ III] $/ \mathrm{H} \alpha$ versus $\left[\mathrm{N}_{\mathrm{II}}\right] /\left[\mathrm{O}_{\mathrm{II}}\right]$ diagnostic diagram. Following the results discussed in Section 6, this plane is able to mitigate the metallicity degeneracy. Since $\left[\mathrm{O}_{\mathrm{III}}\right] / \mathrm{H} \alpha$ and $\left[\mathrm{N}_{\mathrm{II}}\right] /\left[\mathrm{O}_{\mathrm{II}}\right]$ are affected by dust extinction, we corrected the involved emission lines adopting the Calzetti extinction curve (Calzetti et al. 2000) and using the $\mathrm{H} \alpha / \mathrm{H} \beta$ ratio to estimate the nebular colour excess $E(B-V)$. It is worth noting that, since the metallicity degeneracy strongly affects the $\left[\mathrm{O}_{\mathrm{III}}\right] / \mathrm{H} \beta$ versus $[\mathrm{N} I I] / \mathrm{H} \alpha$ plane, we do not use it to identify possible quenching candidates, although all the objects ly-

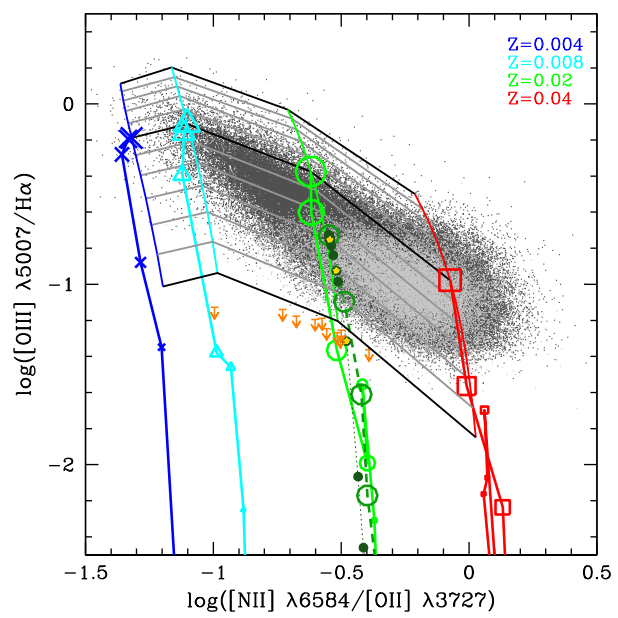

Figure 16. Quenching candidates within the $\left[\mathrm{O}_{\mathrm{III}}\right] / \mathrm{H} \alpha$ versus $[\mathrm{N}$ II $] /[\mathrm{O}$ II $]$ plane. Dark grey points are galaxies extracted from the SDSS DR8 with $\mathrm{S} / \mathrm{N}(\mathrm{H} \alpha)>5, \mathrm{~S} / \mathrm{N}(\mathrm{H} \beta)>3$ and $\mathrm{S} / \mathrm{N}\left(\left[\mathrm{N}_{\mathrm{II}}\right]\right), \mathrm{S} / \mathrm{N}([\mathrm{O} \mathrm{II}])$ and $\mathrm{S} / \mathrm{N}\left(\left[\mathrm{O}_{\mathrm{II}}\right]\right)>2$, while light grey points are galaxies with $\mathrm{S} / \mathrm{N}([\mathrm{O} \mathrm{III}])<2$. The superimposed grid is our set of fixed-age models with different metallicities, as in Fig. 3. Coloured curves associated with different symbols are evolving-age models with an initial $\log (U)_{0}=-3$ (with symbol size decreasing for increasing mass) for the four considered metallicities and with a time-step of $1 \mathrm{Myr}$. For $Z=0.02$, evolving-age models obtained for the truncated (dark green empty circles) and the exponentially declining (dark green filled circles) SFHs are shown with a time-step of $1 \mathrm{Myr}$ within the first $10 \mathrm{Myr}$ after quenching, $\sim 20 \mathrm{Myr}$ from 10 to $100 \mathrm{Myr}$ after quenching, and $100 \mathrm{Myr}$ even further. For the exponentially declining SFH, small gold stars mark the values of the emission line ratios corresponding to 10, 80 and $200 \mathrm{Myr}$ after the SF quenching, from top to bottom. Orange downward arrows are the 10 extreme quenching candidates with $\mathrm{S} / \mathrm{N}\left(\left[\mathrm{O}_{\mathrm{III}}\right]\right)<2$.

ing below $\log ([\mathrm{O}$ III $] / \mathrm{H} \beta)<-0.5$ would fulfil our selection criterion (see Fig. 3).

As it is possible to note from Fig. 16, the bulk of the data distribution lies within a range of fixed-age ionization parameters going from $\log (U)_{0}=-3$ to $\log (U)_{0}=-3.6$, and spans the entire metallicity range of our models (the discrepancy between data and models at high values of $[\mathrm{NII}] /[\mathrm{O} I \mathrm{II}]$ can be explained as in Section 4.1). The median of the data distribution can be described by $-3.2 \lesssim$ $\log (U)_{0} \lesssim-3$ decreasing for increasing metallicity, in agreement with the literature results concerning star-forming galaxies at low redshifts (e.g. Dopita et al. 2006; Nakajima \& Ouchi 2014; Shirazi, Brinchmann \& Rahmati 2014; Hayashi et al. 2015; Kashino et al. 2016; Onodera et al. 2016). Given this latter consideration, we also show evolving-age models characterized by $\log (U)_{0}=-3$ at the time in which the SF stops, both in the SSP and the smoother SFH cases (see Section 5.4), for $Z=0.02$. We find that the initial value of the $[\mathrm{O} I I] / H \alpha$ ratio is lower for smoother SFHs than for SSPs, as already discussed in Section 5.4. Furthermore, for the exponentially declining $\mathrm{SFH}$, the decrease of $[\mathrm{O} \mathrm{III}] / \mathrm{H} \alpha$ is very slow, with the $\left[\mathrm{O}_{\mathrm{III}}\right] / \mathrm{H} \alpha$ values corresponding to the first $10 \mathrm{Myr}$ after the SF quenching accumulating within $\lesssim 0.1$ dex.

Since within this diagnostic diagram metallicity effects are separated from ionization ones, at each $\left[\mathrm{N}_{\mathrm{II}}\right] /[\mathrm{O}$ II] (i.e. at each metallicity), objects with the lowest $[\mathrm{O} \mathrm{III}] / \mathrm{H} \alpha$ ratios could be considered as quenching candidates. Starting from this, we select a subsample of 10 extreme objects with $[\mathrm{O} \mathrm{III}] / \mathrm{H} \alpha$ below the values corresponding to the lowest $\log (U)_{0}=-3.6$ and with $\mathrm{S} / \mathrm{N}([\mathrm{O}$ III $])<2$. This ionization level is consistent with the quiescent phase of SSP evolving-age models, reached $\sim 2$ Myr after quenching and 


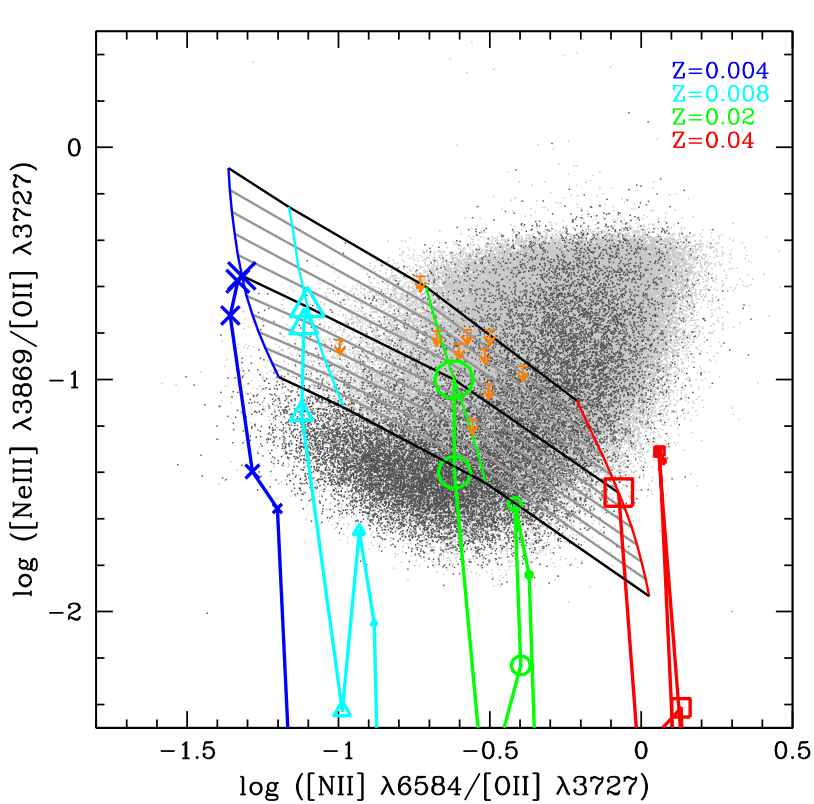

Figure 17. Quenching candidates within the $\left[\mathrm{Ne}\right.$ III]/[O II] versus $\left[\mathrm{N}_{\text {II }}\right] /[\mathrm{O}$ II] plane. Dark grey points are galaxies extracted from the SDSS DR8 with $\mathrm{S} / \mathrm{N}(\mathrm{H} \alpha)>5, \mathrm{~S} / \mathrm{N}(\mathrm{H} \beta)>3$, and $\mathrm{S} / \mathrm{N}\left(\left[\mathrm{N}_{\mathrm{II}}\right]\right), \mathrm{S} / \mathrm{N}([\mathrm{O} \mathrm{II}]), \mathrm{S} / \mathrm{N}([\mathrm{Ne} \mathrm{III}])>2$, while light grey points are galaxies with $\mathrm{S} / \mathrm{N}([\mathrm{Ne} \mathrm{III}])<2$. Colours and symbols are defined as in Fig. 3. Orange downward arrows are the 10 extreme quenching candidates with $\mathrm{S} / \mathrm{N}\left(\left[\mathrm{O}_{\text {III }}\right]\right)<2$.

corresponding to $\log (U)_{t} \sim-3.2$. If smoother SFHs are assumed, these very low levels of $[\mathrm{O} \mathrm{III}] / \mathrm{H} \alpha$ are instead reached $\sim 200 \mathrm{Myr}$ after the SF quenching, at $\log (U)_{t} \sim-3.4$.

The 10 selected objects are not detected in [O III] and they have upper limits $\lesssim-1.1$ on the $\mathrm{O}$ III/ $\mathrm{H} \alpha$ ratio. Moreover, based on our models of $[\mathrm{N}$ II]/[O II] and on Tremonti et al. (2004) models, they have intermediate metallicities [i.e. $\log \left([\mathrm{N}\right.$ II $\left.] /\left[\mathrm{O}_{\mathrm{II}}\right]\right)$ ranging from $\sim-1$ to $\sim-0.4]$, and a faint $[\mathrm{Ne}$ III] emission line with $\mathrm{S} / \mathrm{N}([\mathrm{Ne} \mathrm{III}])<2$ (see Section 4.1). This is compatible with our suggestion that galaxies that have quenched their SF in the recent past can be characterized by the absence of high-ionization lines. Moreover, it confirms that the two proposed emission line ratios are complementary in the identification of quenching candidates.

Fig. 17 illustrates the 10 objects in the $[\mathrm{Ne}$ $[\mathrm{N}$ II $] /[\mathrm{O}$ II $]$ diagnostic diagram. Within this plane, the candidates have apparently high $[\mathrm{Ne} I I I] /[\mathrm{O} I I]$ ratios but, since $[\mathrm{Ne} I I I]$ is undetected, these values are actually upper limits, thus the real $[\mathrm{Ne}$ III $] /[\mathrm{O}$ II] ratios of the 10 objects can be much lower than the illustrated ones. The same holds for all the objects lying in the upper right or outside the model grid in Fig. 17, which are characterized by $2<\mathrm{S} / \mathrm{N}([\mathrm{Ne} \mathrm{III}])<4$.

\subsection{Properties of the quenching candidates}

In this section we illustrate the main properties of the 10 selected quenching galaxies, verifying if they are compatible with a recent quenching of the SF.

Figs 18 and 19 illustrate the candidate spectra, corrected for dust extinction adopting the Calzetti extinction curve (Calzetti et al. 2000) and the $\mathrm{H} \alpha / \mathrm{H} \beta$ ratio to estimate the nebular colour excess $E(B-V)$. In particular, we applied the same correction for both the continuum and the emission lines. Spectra lack strong absorption Balmer lines, which are characteristic of the post-starburst phase.
This indicates that the candidates may have been caught in an earlier evolutionary phase with respect to the post-starburst (E+A, K+A) one (e.g. Dressler \& Gunn 1983; Couch \& Sharples 1987; Poggianti et al. 2008). Moreover, they have strong $\mathrm{H} \alpha$ and [O II] lines, in agreement with our predictions that low-ionization lines decrease by a small factor with respect to high-ionization ones in just quenched objects (cf. Section 5.1). Fig. 19 shows the median stacked spectrum of the 10 candidates obtained by stacking the individual spectra before and after applying the correction for dust extinction. In this latter case we assumed the Calzetti et al. (2000) extinction curve and the same nebular colour excess $E(B-V)$ for both emission lines and continuum. The 10 candidates have $0.2 \lesssim E(B-V) \lesssim$ 0.8 , with a mean $E(B-V) \sim 0.5 \pm 0.2$. The large $E(B-V)$ of some of them explains why the stacked spectrum derived from individual spectra corrected for dust extinction is significantly bluer than the one derived from dust-extincted spectra. We find that, even after the stacking, which increases the S/N of the individual spectra, the stacked spectrum has very faint high-ionization lines in both cases. This confirms that the absence of the high-ionization emission lines is a real feature and not an artefact due to low signal in the individual spectra.

The 10 candidates have high $\mathrm{H} \alpha$ luminosities $L(\mathrm{H} \alpha)$ (as expected from the evolution described in Section 5.1), ranging from $10^{40}$ to $10^{42} \mathrm{erg} \mathrm{s}^{-1} \AA^{-1}$, and a median SFR $\sim 1.4 \pm 1.7 \mathrm{M}_{\odot} \mathrm{yr}^{-1}$. However, we remind that the usual recipes to convert $L(\mathrm{H} \alpha)$ into SFR cannot be applied to our galaxies because most $\mathrm{O}$ stars are missing compared to a galaxy where SF is still ongoing. Therefore, the $L(\mathrm{H} \alpha)$-derived SFRs represent the past SFRs (i.e. the ones before the quenching) and thus should be considered as upper limits. We also find that the 10 candidates have elliptical and S0 morphologies. We refer to the companion paper Quai et al. (in preparation) for a detailed discussion about the typical morphologies of complete samples of quenching candidates and how they relate to the parent population of SF galaxies.

The nature of the 10 quenching candidates is also shown in Fig. 20 , which illustrates the $(u-r)$-mass diagram for our sample. Following Schawinski et al. (2014), we corrected colours for dust extinction assuming the colour excess of the stellar continuum, defined as $E_{s}(B-V)=0.44 \times E(B-V)$, where $E(B-V)$ is the nebular continuum adopted so far (Calzetti et al. 2000). Colours are compatible with the evolution discussed in Section 5.5 at early times after the SF halt, and are bluer than the green valley $(u-r)$ colours (corrected for dust extinction) derived by Schawinski et al. (2014). This suggests that the 10 galaxies may be at the beginning of the quenching process, not being yet as red as green valley galaxies. Furthermore, it is interesting to note that they have $\log \left(\mathrm{M} / \mathrm{M}_{\odot}\right) \sim 10$, compatible with the mass of the passive galaxy population building up at low-intermediate redshifts (e.g. Pozzetti et al. 2010).

Since the $\left[\mathrm{O}_{\mathrm{III}}\right] / \mathrm{H} \alpha$ and the $[\mathrm{N}$ II $] /\left[\mathrm{O}_{\mathrm{II}}\right]$ emission line ratios are affected by dust extinction, we verified if our results are influenced by the choice of the extinction law assumed to correct our data. We adopt the Allen (1976) extinction curve, comparing the results with the ones obtained so far using the Calzetti et al. (2000) one. We quantify the effect of the two extinction laws on the emission line ratios $[\mathrm{O}$ III $] / \mathrm{H} \alpha$ and $[\mathrm{N} \mathrm{II}] /[\mathrm{O}$ II] by calculating the median extinction differences $\left(A_{[\mathrm{N} \mathrm{II}]}-A_{[\mathrm{O} I \mathrm{II}]}\right)$ and $\left(A_{[\mathrm{O} \mathrm{III}]}-A_{\mathrm{H} \alpha}\right)$ for both cases. We find that $\left(A_{[\mathrm{OIII}]}-A_{\mathrm{H} \alpha}\right)$ is $\sim 0.35$ in both cases, while $\left(A_{[\mathrm{NII}]}-\right.$ $A_{[\mathrm{O} \text { II }]}$ ) differs by $\sim 0.03 \mathrm{mag}$, with the Allen (1976) extinction curve producing slightly higher $[\mathrm{N} \Pi] /\left[\mathrm{O}_{\Pi}\right]$. This very small discrepancy leads us to conclude that our approach is not influenced by the choice of the extinction curve. 


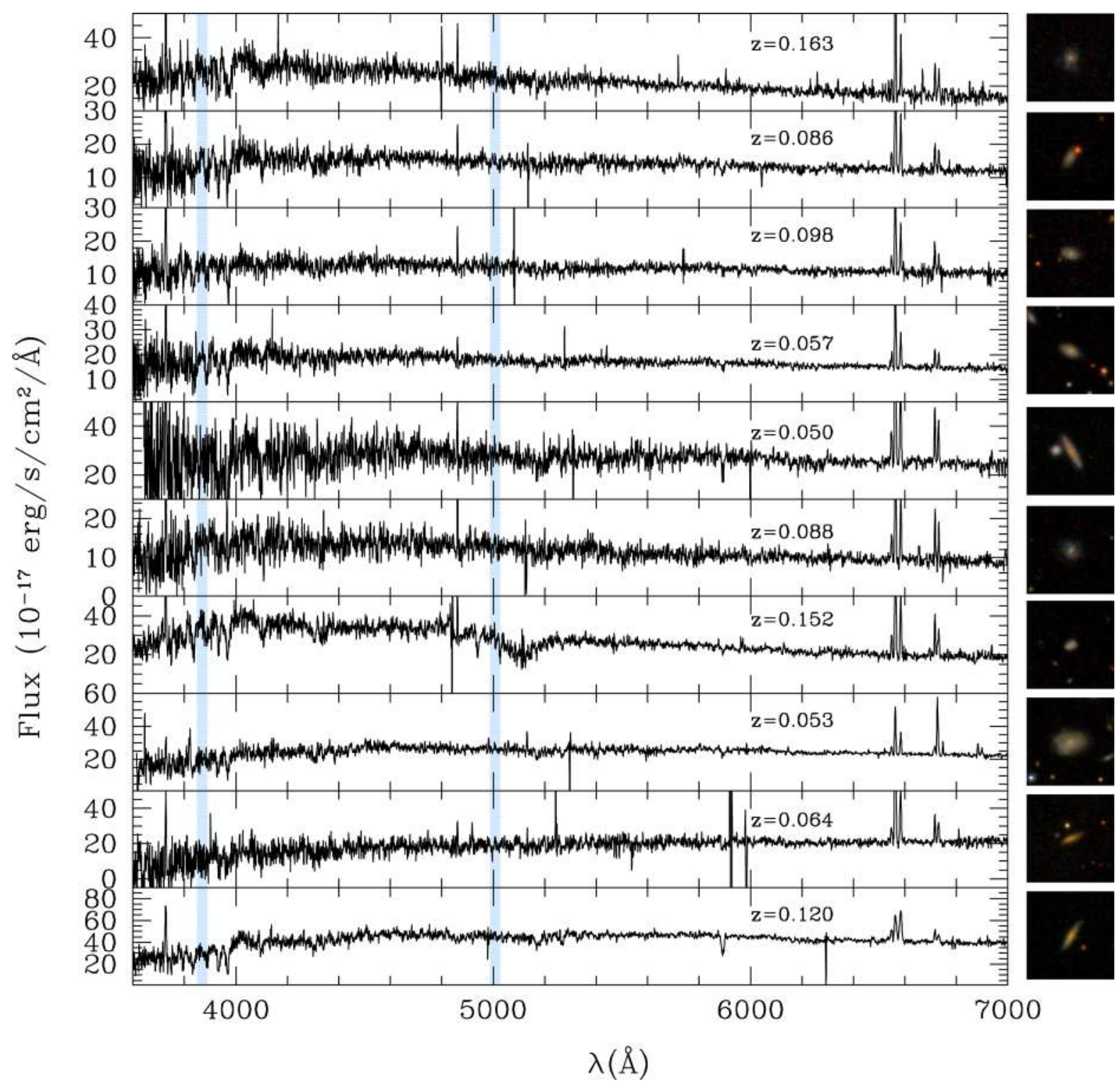

Figure 18. Spectra of the 10 extreme quenching candidates corrected for dust extinction using the nebular colour excess $E(B-V)$. The black curves are spectra corrected for dust extinction. The emission lines discussed in this work are indicated on the top of the figure (from left to right: [O III], [Ne III], [O III], $\mathrm{H} \alpha,[\mathrm{N} I I]$ and $\left[\mathrm{S} \mathrm{II}_{\text {II }}\right.$, and light blue shaded regions mark the not-detected [Ne III] and [O III] lines. Redshifts are reported for each object and morphologies are shown on the right side of each spectrum.

We also investigated if our results are valid when dust-free diagnostic diagrams are used, which would have the advantage of producing results not relying on the assumption of an extinction law. Fig. 21 shows the 10 quenching candidates within the $[\mathrm{O}$ III $] / \mathrm{H} \beta$ versus $\left[\mathrm{N}_{\mathrm{II}}\right] /\left[\mathrm{S}_{\mathrm{II}}\right]$ plane, which involves ratios between emission lines closer in wavelength, and thus is basically unaffected by dust extinction. Although the discrepancy between models and data at the highest metallicities is still present, within this plane the 10 galaxies are located at the lowest edge of the data distribution, suggesting that the $[\mathrm{O}$ III $] / \mathrm{H} \beta$ can be used as a dust-free, quenching diagnostic, similarly to $[\mathrm{O}$ III $] / \mathrm{H} \alpha$. However, the $\left[\mathrm{N}\right.$ II] $/\left[\mathrm{S}_{\mathrm{II}}\right]$ ratio, although being appropriate to avoid the uncertainties related to dust extinction, involves weaker lines like [S $\left.\mathrm{SI}_{\mathrm{I}}\right]$ and is less efficient in separating different metallicities compared to $[\mathrm{N} \mathrm{II}] /[\mathrm{O} \mathrm{II}]$, also producing larger errors relative to the covered range in metallicity.
Since the 10 selected objects are just an example of quenching candidates, they do not represent a complete sample of just quenched galaxies and cannot be used to derive reliable quenching candidate fractions (see Section 5.6). We refer to Quai et al. (in preparation) for a detailed discussion about them.

\section{CONCLUSIONS}

In this work, we propose a methodology aimed at identifying galaxies in the phase which immediately follows the quenching of the SF, under the assumption of a fast quenching (shorter than $200 \mathrm{Myr}$ ), both for an extreme sharp case and for a more realistic, smoother SF decline. Our approach is based on the use of specific emission line ratios involving high- and low-ionization lines, which are expected to be strongly affected by quenching, rapidly dropping when the 


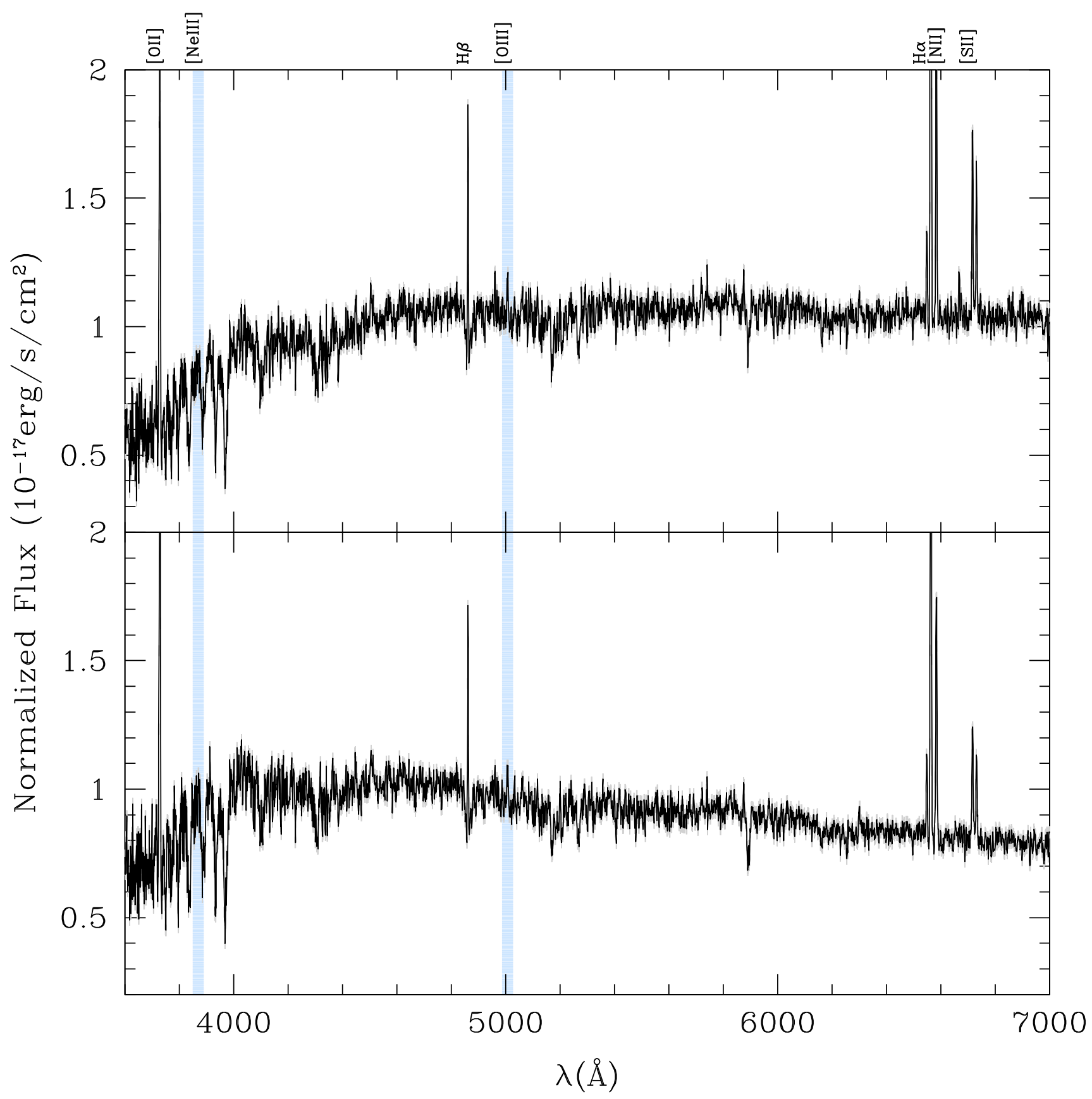

Figure 19. Median stacked spectrum of the 10 extreme quenching candidates. The top panel illustrates the median stacked spectrum (black curve) obtained from the dust-extincted quenching candidate spectra. The bottom panel shows the median stacked spectrum (black curve) obtained correcting the quenching candidate spectra for dust extinction, adopting the nebular $E(B-V)$ (Calzetti et al. 2000) for both continuum and emission lines. Errors are shown in grey. The emission lines discussed in this work are indicated on the top of the figure (from left to right: [O II], [Ne III], [O III], $\mathrm{H} \alpha$, [N II] and [S II]). Light blue shaded regions mark the not-detected [Ne III] and [O III] lines.

most massive $\mathrm{O}$ stars able to produce high-ionization lines disappear. Our main findings can be summarized as follows.

(i) We focus in particular on the $[\mathrm{O}$ III] $] / \mathrm{H} \alpha$ and $[\mathrm{Ne}$ III $] /[\mathrm{O}$ II] emission line ratios, modelling them by means of the CLOUDY (Ferland et al. 2013) photoionization code. We find that they are able to trace the phase just after the SF quenching, decreasing even by more than a factor of 10 after its occurrence. The time-scales of this decline depend on the SFH adopted to characterize the ionizing stellar population. In particular, if the extreme case of a sharp quenching is assumed, the emission line ratios definitively drop on time-scales of $\sim 10$ Myr after the SF quenching, while, if a smoother and more realistic quenching is considered (e.g. an exponentially declining SFR with an $e$-folding time $\tau=200 \mathrm{Myr}$ ) they decline by a factor of $\sim 2$ within the time interval needed by the star-forming region to become quiescent (i.e. $\sim 80 \mathrm{Myr}$ ).

(ii) Our methodology is valid even when different synthetic stellar spectra are assumed to characterize the central ionizing source. In particular, in the case of a sharp quenching, we find a good agreement between STARBURST 99 and BC03 models: the decline of 


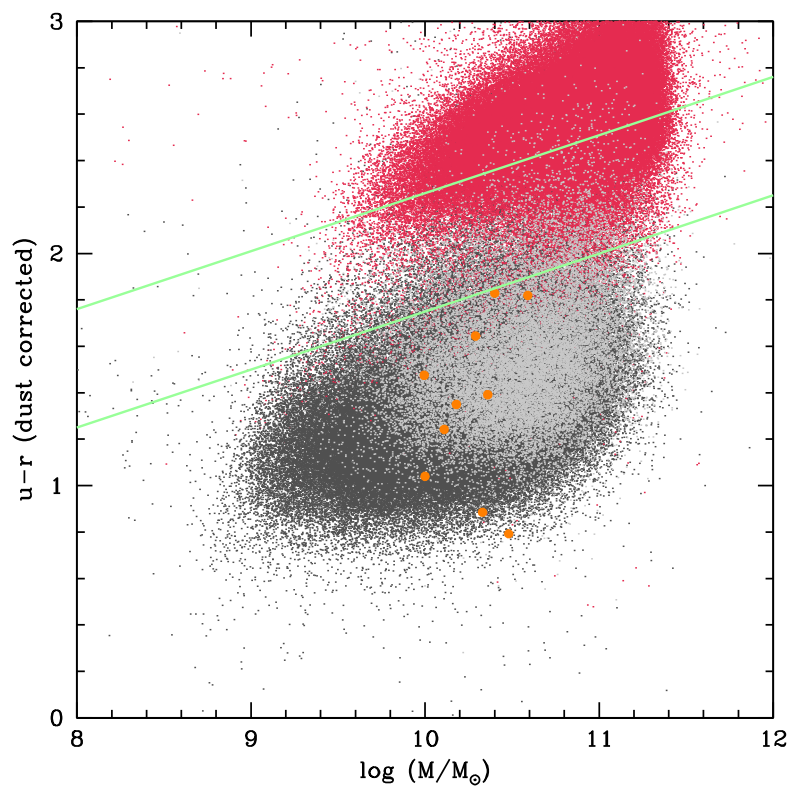

Figure 20. Colour-mass diagram for our galaxy sample $[(u-r)$ colours are corrected for dust extinction]. Dark grey points are galaxies extracted from the SDSS DR8 with $\mathrm{S} / \mathrm{N}(\mathrm{H} \alpha)>5, \mathrm{~S} / \mathrm{N}(\mathrm{H} \beta)>3$ and with $\mathrm{S} / \mathrm{N}\left(\left[\mathrm{N}_{\mathrm{II}}\right]\right)$, $\mathrm{S} / \mathrm{N}([\mathrm{O} \mathrm{II}])$ and $\mathrm{S} / \mathrm{N}([\mathrm{O} \mathrm{II}])>2$, while light grey points are galaxies with $\mathrm{S} / \mathrm{N}\left(\left[\mathrm{O}_{\mathrm{III}}\right]\right)<2$. Red points are galaxies with $\mathrm{S} / \mathrm{N}(\mathrm{H} \alpha)<5$ and/or $\mathrm{EW}(\mathrm{H} \alpha)>0$ (see Quai et al., in preparation for further details). Orange circles are the 10 extreme quenching candidates with $\mathrm{S} / \mathrm{N}\left(\left[\mathrm{O}_{\text {III }}\right]\right)<2$. Green lines mark the green valley defined by Schawinski et al. (2014). This is taken as reference since it was derived from a sample of low-redshift galaxies, as ours.

the two emission line ratios occurs in both cases and happens on similar time-scales, at each metallicity. This is an interesting result, since BC03 models are generally used to describe more advanced phases of galaxy evolution.

(iii) We find that our approach is influenced by the ionizationmetallicity degeneracy, since more metallic ionizing sources, being characterized by a low number of hard UV ionizing photons, can produce the same low values of the analysed emission line ratios as quenching ionizing sources. We find that this degeneracy can be reduced using pairs of independent emission line ratios, separately related to metallicity and ionization. In particular, due to its strong dependence on metallicity and weak dependence on ionization parameter, we propose the $[\mathrm{N} I I] /\left[\mathrm{O}_{\mathrm{II}}\right]$ ratio as metallicity diagnostic to mitigate the degeneracy, and alternatively the $[\mathrm{N}$ II $] /\left[\mathrm{S}_{\mathrm{II}}\right]$ one, less affected by dust attenuation but involving weaker lines like [S II].

(iv) We compare our models to a sample of $\sim 174000$ SDSS DR8 star-forming galaxies in order to verify if objects characterized by low values of $\left[\mathrm{O}_{\mathrm{III}}\right] / \mathrm{H} \alpha$ and $[\mathrm{Ne}$ III $] /\left[\mathrm{O}_{\mathrm{II}}\right]$ are indeed present in the global galaxy population and if their properties are compatible with a recent $\mathrm{SF}$ quenching. We mainly use the $[\mathrm{O}$ III $] / \mathrm{H} \alpha$ versus $[\mathrm{N}$ II $] /\left[\mathrm{O}_{\mathrm{II}}\right]$ diagnostic diagram, since it is able to separate metallicity effects from ionization ones. Within this plane, we identify 10 objects with extremely low $[\mathrm{O}$ III $] / \mathrm{H} \alpha$ ratios and $\mathrm{S} / \mathrm{N}\left(\left[\mathrm{O}_{\mathrm{III}}\right]\right)<2$. We find that their spectra lack of the [Ne III] line, but are characterized by rather stron $\mathrm{H} \alpha$ and [O II] lines. All these properties agree with the hypothesis that they may have quenched their SF in the recent past;

(v) Within the dust-corrected $(u-r)$-mass diagram, 10 candidates lie outside the green valley, due to the bluer colours. These latter are compatible with the evolution predicted by our models

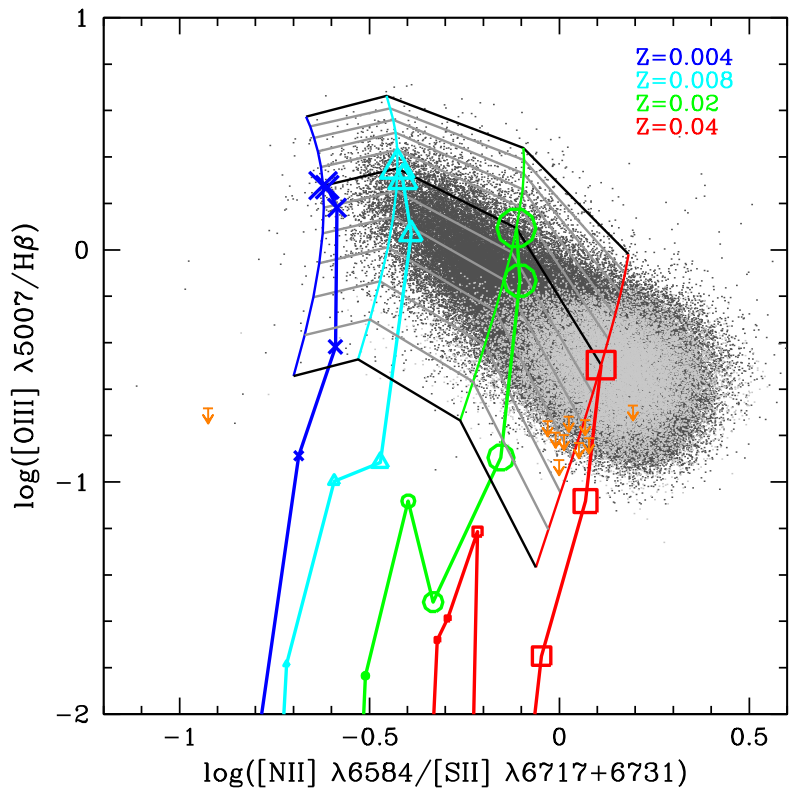

Figure 21. Quenching candidates within the $[\mathrm{O} I I] / H \beta$ versus $\left[\mathrm{N}_{\mathrm{II}}\right] /[\mathrm{S}$ II] plane. Dark grey points are galaxies extracted from the SDSS DR8 with $\mathrm{S} / \mathrm{N}(\mathrm{H} \beta)>3$ and $\mathrm{S} / \mathrm{N}([\mathrm{N} I]), \mathrm{S} / \mathrm{N}([\mathrm{S} I]), \mathrm{S} / \mathrm{N}\left(\left[\mathrm{O}_{\mathrm{III}}\right]\right)>2$, while light grey points are galaxies with $\mathrm{S} / \mathrm{N}([\mathrm{O}$ III] $)<2$. Colours and symbols of the grid are defined as in Fig. 3. Orange arrows are the 10 extreme quenching candidates with $\mathrm{S} / \mathrm{N}([\mathrm{O}$ III] $)<2$.

after 10-100 Myr from the SF quenching, further suggesting that the 10 objects may be at the beginning of the quenching process;

(vi) We find that our methodology is independent from the dust extinction curve adopted to correct the data. Moreover, the $[\mathrm{O}$ III $] / \mathrm{H} \beta$ ratio is similar to $[\mathrm{O} \mathrm{III}] / \mathrm{H} \alpha$ ratio in identifying quenching candidates, while $[\mathrm{N} \mathrm{II}] /[\mathrm{S} \mathrm{II}]$, although being unaffected by dust extinction, has the disadvantage of involving the weaker [S II] line and of being less efficient in separating metallicities with respect to $[\mathrm{N}$ II $] /\left[\mathrm{O}_{\mathrm{II}}\right]$, also producing larger errors relative to the covered range in metallicity.

(vii) We verify that our approach is valid also when dust-free diagnostic diagrams, such as the $\left[\mathrm{O}_{\mathrm{III}}\right] / \mathrm{H} \beta$ versus $\left[\mathrm{N}_{I I}\right] /\left[\mathrm{S}_{\mathrm{II}}\right]$ one, are used. Indeed, the 10 quenching candidates lie at the lowest edge of the data distribution also in this plane.

The proposed methodology suggests that emission line ratios involving high- and low-ionization lines are powerful tools to identify galaxies in the quenching phase. Moreover, this kind of approach has the advantage of being applicable also at higher redshifts, once that suitable pairs of emission lines are chosen. Therefore, our methodology can be used for the analysis of large spectroscopic surveys data such as JWST (Gardner et al. 2006), WFIRST (Spergel et al. 2013) and Euclid (Laureijs et al. 2011), which identify a large number of $\mathrm{H} \alpha$ emitters at $z>1$ (e.g. Pozzetti et al. 2016). Since the 10 candidates identified in this work are just examples of quenching galaxies, they cannot be used to derive reliable quenching fractions. However, our results indicate that once a complete sample of quenching candidates is identified within an SF galaxy sample, their observed fraction, compared to the global population of starforming galaxies, could be used to disentangle what is the quenching mechanism at work and what are its typical time-scales. We refer to a companion paper (Quai et al., in preparation) both for this purpose and for the investigation of the detailed properties of complete samples of quenching candidates. 


\section{ACKNOWLEDGEMENTS}

The authors are grateful to Emanuele Daddi and Grażyna Stasińska for useful discussion. ACit is also grateful to Lucia Armillotta, Alfonso Veropalumbo and Gianni Zamorani for helpful discussion and suggestions. The authors also acknowledge the support of the grant PRIN MIUR 2010-2011 The dark Universe and the cosmic evolution of baryons: from current surveys to Euclid, PRIN MIUR 2015 Cosmology and Fundamental Physics: illuminating the Dark Universe with Euclid and the grant ASI n.I/023/12/0 Attivitrelative alla fase B2/C per la missione Euclid. Funding for the SDSS and SDSS-II has been provided by the Alfred P. Sloan Foundation, the Participating Institutions, the National Science Foundation, the U.S. Department of Energy, the National Aeronautics and Space Administration, the Japanese Monbukagakusho, the Max Planck Society, and the Higher Education Funding Council for England. The SDSS Web Site is http://www.sdss.org/. The SDSS is managed by the Astrophysical Research Consortium for the Participating Institutions. The Participating Institutions are the American Museum of Natural History, Astrophysical Institute Potsdam, University of Basel, University of Cambridge, Case Western Reserve University, University of Chicago, Drexel University, Fermilab, the Institute for Advanced Study, the Japan Participation Group, Johns Hopkins University, the Joint Institute for Nuclear Astrophysics, the Kavli Institute for Particle Astrophysics and Cosmology, the Korean Scientist Group, the Chinese Academy of Sciences (LAMOST), Los Alamos National Laboratory, the Max-Planck-Institute for Astronomy (MPIA), the Max-Planck-Institute for Astrophysics (MPA), New Mexico State University, Ohio State University, University of Pittsburgh, University of Portsmouth, Princeton University, the United States Naval Observatory and the University of Washington.

\section{REFERENCES}

Allen C. W., 1976, Astrophysical Quantities, 3rd edn. Athlone, London Asplund M., Grevesse N., Sauval A. J., 2005, in Barnes T. G., III, Bash F. N., eds, ASP Conf. Ser. Vol. 336, Cosmic Abundances as Records of Stellar Evolution and Nucleosynthesis. Astron. Soc. Pac., San Francisco, p. 25

Baldry I. K., Glazebrook K., Brinkmann J., Ivezić Ž., Lupton R. H., Nichol R. C., Szalay A. S., 2004, ApJ, 600, 681

Baldwin J. A., Phillips M. M., Terlevich R., 1981, PASP, 93, 5

Balogh M. L. et al., 2011, MNRAS, 412, 2303

Bekki K., 1998, ApJ, 502, L133

Bekki K., Couch W. J., Shioya Y., 2002, ApJ, 577, 651

Bell E. F., de Jong R. S., 2001, ApJ, 550, 212

Bell E. F. et al., 2004, ApJ, 600, L11

Bell E. F. et al., 2012, ApJ, 753, 167

Benson A. J., 2012, New Astron., 17, 175

Blanton M. R. et al., 2003, ApJ, 594, 186

Bournaud F., Jog C. J., Combes F., 2007, A\&A, 476, 1179

Boylan-Kolchin M., Springel V., White S. D. M., Jenkins A., Lemson G., 2009, MNRAS, 398, 1150

Brammer G. B. et al., 2009, ApJ, 706, L173

Brammer G. B. et al., 2011, ApJ, 739, 24

Bressan A., Chiosi C., Fagotto F., 1994, ApJS, 94, 63

Brinchmann J., Charlot S., White S. D. M., Tremonti C., Kauffmann G., Heckman T., Brinkmann J., 2004, MNRAS, 351, 1151

Brinchmann J., Charlot S., Kauffmann G., Heckman T., White S. D. M., Tremonti C., 2013, MNRAS, 432, 2112

Bruzual G., Charlot S., 2003, MNRAS, 344, 1000

Calzetti D., Armus L., Bohlin R. C., Kinney A. L., Koornneef J., StorchiBergmann T., 2000, ApJ, 533, 682

Carpineti A., Kaviraj S., Darg D., Lintott C., Schawinski K., Shabala S., 2012, MNRAS, 420, 2139

Cassata P. et al., 2008, A\&A, 483, L39
Charlot S., Longhetti M., 2001, MNRAS, 323, 887

Chen X. Y., Liang Y. C., Hammer F., Prugniel P., Zhong G. H., Rodrigues M., Zhao Y. H., Flores H., 2010, A\&A, 515, A101

Cheung E. et al., 2012, ApJ, 760, 131

Cicone C. et al., 2014, A\&A, 562, A21

Ciesla L. et al., 2016, A\&A, 585, A43

Cimatti A. et al., 2013, ApJ, 779, L13

Citro A., Pozzetti L., Moresco M., Cimatti A., 2016, A\&A, 592, A19

Couch W. J., Sharples R. M., 1987, MNRAS, 229, 423

Courteau S., de Jong R. S., Broeils A. H., 1996, ApJ, 457, L73

da Cunha E., Eminian C., Charlot S., Blaizot J., 2010, MNRAS, 403, 1894

Dasyra K. M. et al., 2006, ApJ, 651, 835

Debattista V. P., Mayer L., Carollo C. M., Moore B., Wadsley J., Quinn T., 2006, ApJ, 645, 209

Dekel A., Birnboim Y., 2006, MNRAS, 368, 2

Denicoló G., Terlevich R., Terlevich E., 2002, MNRAS, 330, 69

Diamond-Stanic A. M., Moustakas J., Tremonti C. A., Coil A. L., Hickox R. C., Robaina A. R., Rudnick G. H., Sell P. H., 2012, ApJ, 755, L26

Dopita M. A., Kewley L. J., Heisler C. A., Sutherland R. S., 2000, ApJ, 542, 224

Dopita M. A. et al., 2006, ApJS, 167, 177

Draine B. T. et al., 2007, ApJ, 663, 866

Dressler A., Gunn J. E., 1983, ApJ, 270, 7

Drory N., Bender R., Feulner G., Hopp U., Maraston C., Snigula J., Hill G. J., 2004, ApJ, 608, 742

Eisenstein D. J. et al., 2011, AJ, 142, 72

Faber S. M., Huang J.-S., Noeske K. G., Bundy K., DEEP2 Team IRAC GTO Team Palomar K-band Team, 2005, American Astronomical Society Meeting Abstracts. p. 1298

Fabian A. C., 2012, ARA\&A, 50, 455

Farouki R., Shapiro S. L., 1981, ApJ, 243, 32

Ferland G. J., Korista K. T., Verner D. A., Ferguson J. W., Kingdon J. B., Verner E. M., 1998, PASP, 110, 761

Ferland G. J. et al., 2013, Rev. Mex. Astron. Astrofis., 49, 137

Förster Schreiber N. M. et al., 2014, ApJ, 787, 38

Fraser-McKelvie A., Brown M. J. I., Pimbblet K. A., Dolley T., Crossett J. P., Bonne N. J., 2016, MNRAS, 462, L11

Gardner J. P. et al., 2006, Space Sci. Rev., 123, 485

Genzel R., Tacconi L. J., Rigopoulou D., Lutz D., Tecza M., 2001, ApJ, 563,527

Genzel R. et al., 2014, ApJ, 785, 75

Greis S. M. L., Stanway E. R., Davies L. J. M., Levan A. J., 2016, MNRAS, 459,2591

Gunn J. E., Gott III J. R., 1972, ApJ, 176, 1

Guzmán R., Gallego J., Koo D. C., Phillips A. C., Lowenthal J. D., Faber S. M., Illingworth G. D., Vogt N. P., 1997, ApJ, 489, 559

Hayashi M. et al., 2015, PASJ, 67, 80

Hibbard J. E., van Gorkom J. H., 1996, AJ, 111, 655

Hillier D. J., Miller D. L., 1998, ApJ, 496, 407

Hopkins P. F., Hernquist L., Cox T. J., Di Matteo T., Martini P., Robertson B., Springel V., 2005, ApJ, 630, 705

Issa M. R., MacLaren I., Wolfendale A. W., 1990, A\&A, 236, 237

Johansson J., Thomas D., Maraston C., 2012, MNRAS, 421, 1908

Juarez Y., Maiolino R., Mujica R., Pedani M., Marinoni S., Nagao T., Marconi A., Oliva E., 2009, A\&A, 494, L25

Karim A. et al., 2011, ApJ, 730, 61

Kashino D. et al., 2016, preprint (arXiv:1604.06802)

Kauffmann G. et al., 2003a, MNRAS, 341, 54

Kauffmann G. et al., 2003b, MNRAS, 346, 1055

Kaviraj S., 2010, MNRAS, 408, 170

Kaviraj S., Kirkby L. A., Silk J., Sarzi M., 2007, MNRAS, 382, 960

Kewley L. J., Dopita M. A., 2002, ApJS, 142, 35

Kewley L. J., Dopita M. A., Sutherland R. S., Heisler C. A., Trevena J., 2001, ApJ, 556, 121 (Kew01)

Kewley L. J., Dopita M. A., Leitherer C., Davé R., Yuan T., Allen M., Groves B., Sutherland R., 2013, ApJ, 774, 100

Khochfar S., Silk J., 2006, ApJ, 648, L21

Kormendy J., Kennicutt Jr R. C., 2004, ARA\&A, 42, 603 
Larson R. B., Tinsley B. M., Caldwell C. N., 1980, ApJ, 237, 692

Laureijs R. et al., 2011, preprint (arXiv:1110.3193)

Leitherer C. et al., 1999, ApJS, 123, 3

Lejeune T., Cuisinier F., Buser R., 1997, A\&AS, 125

Levesque E. M., Kewley L. J., Larson K. L., 2010, AJ, 139, 712 (Lev10)

Lisenfeld U., Ferrara A., 1998, ApJ, 496, 145

Lu Y., Mo H. J., Weinberg M. D., Katz N., 2011, MNRAS, 416, 1949

MacArthur L. A., Courteau S., Holtzman J. A., 2003, ApJ, 582, 689

McIntosh D. H. et al., 2014, MNRAS, 442, 533

Madau P., Dickinson M., 2014, ARA\&A, 52, 415

Madau P., Pozzetti L., Dickinson M., 1998, ApJ, 498, 106

Marsan Z. C. et al., 2015, ApJ, 801, 133

Martig M., Bournaud F., Teyssier R., Dekel A., 2009, ApJ, 707, 250

Martin D. C. et al., 2007, ApJS, 173, 342

Mendel J. T., Simard L., Ellison S. L., Patton D. R., 2013, MNRAS, 429, 2212

Meynet G., Maeder A., Schaller G., Schaerer D., Charbonnel C., 1994, A\&AS, 103, 97

Mobasher B. et al., 2005, ApJ, 635, 832

Mok A. et al., 2013, MNRAS, 431, 1090

Moore B., Lake G., Quinn T., Stadel J., 1999, MNRAS, 304, 465

Morisset C. et al., 2016, A\&A, 594, A37

Moustakas J., Kennicutt Jr R. C., Tremonti C. A., 2006, ApJ, 642, 775

Moustakas J., Kennicutt Jr R. C., Tremonti C. A., Dale D. A., Smith J.-D. T., Calzetti D., 2010, ApJS, 190, 233

Muzzin A. et al., 2012, ApJ, 746, 188

Muzzin A. et al., 2013, ApJS, 206, 8

Naab T., Khochfar S., Burkert A., 2006, ApJ, 636, L81

Naab T., Johansson P. H., Ostriker J. P., Efstathiou G., 2007, ApJ, 658, 710

Naab T., Johansson P. H., Ostriker J. P., 2009, ApJ, 699, L178

Nagao T., Maiolino R., Marconi A., 2006, A\&A, 459, 85

Nakajima K., Ouchi M., 2014, MNRAS, 442, 900

Norman C. A., Sellwood J. A., Hasan H., 1996, ApJ, 462, 114

Onodera M. et al., 2016, ApJ, 822, 42

Pauldrach A. W. A., Hoffmann T. L., Lennon M., 2001, A\&A, 375, 161

Pérez-Montero E. et al., 2013, A\&A, 549, A25

Pettini M., Pagel B. E. J., 2004, MNRAS, 348, L59

Poggianti B. M., Bridges T. J., Komiyama Y., Yagi M., Carter D., Mobasher B., Okamura S., Kashikawa N., 2004, ApJ, 601, 197

Poggianti B. M. et al., 2008, ApJ, 684, 888

Pozzetti L. et al., 2010, A\&A, 523, A13

Pozzetti L. et al., 2016, A\&A, 590, A3

Quilis V., Moore B., Bower R., 2000, Science, 288, 1617

Quintero A. D. et al., 2004, ApJ, 602, 190

Rigby J. R., Rieke G. H., 2004, ApJ, 606, 237
Rothberg B., Joseph R. D., 2004, AJ, 128, 2098

Salim S., 2014, Serbian Astron. J., 189, 1

Sanders D. B., Soifer B. T., Elias J. H., Madore B. F., Matthews K., Neugebauer G., Scoville N. Z., 1988, ApJ, 325, 74

Schaefer A. L. et al., 2017, MNRAS, 464, 121

Schaller G., Schaerer D., Meynet G., Maeder A., 1992, A\&AS, 96, 269

Schawinski K. et al., 2014, MNRAS, 440, 889

Schmutz W., Leitherer C., Gruenwald R., 1992, PASP, 104, 1164

Schweizer F., Seitzer P., 1992, AJ, 104, 1039

Shields J. C., Kennicutt Jr R. C., 1995, ApJ, 454, 807

Shirazi M., Brinchmann J., Rahmati A., 2014, ApJ, 787, 120

Spergel D. et al., 2013, preprint (arXiv:1305.5422)

Springel V. et al., 2005, Nature, 435, 629

Stasińska G., Cid Fernandes R., Mateus A., Sodré L., Asari N. V., 2006, MNRAS, 371, 972

Strateva I. et al., 2001, AJ, 122, 1861

Tal T., van Dokkum P. G., Nelan J., Bezanson R., 2009, AJ, 138, 1417

Taranu D. S., Hudson M. J., Balogh M. L., Smith R. J., Power C., Oman K. A., Krane B., 2014, MNRAS, 440, 1934

Thomas D. et al., 2013, MNRAS, 431, 1383

Tielens A. G. G. M., 2010, The Physics and Chemistry of the Interstellar Medium. Cambridge Univ. Press, Cambridge

Tinker J. L., Wechsler R. H., Zheng Z., 2010, ApJ, 709, 67

Tremonti C. A. et al., 2004, ApJ, 613, 898

van Zee L., Salzer J. J., Haynes M. P., O’Donoghue A. A., Balonek T. J., 1998, AJ, 116, 2805

Veilleux S., Osterbrock D. E., 1987, ApJS, 63, 295

Wetzel A. R., Tinker J. L., Conroy C., van den Bosch F. C., 2013, MNRAS, 432, 336

Whitaker K. E. et al., 2011, ApJ, 735, 86

Whitaker K. E. et al., 2014, ApJ, 795, 104

White S. D. M., Frenk C. S., 1991, ApJ, 379, 52

Wiklind T., Dickinson M., Ferguson H. C., Giavalisco M., Mobasher B., Grogin N. A., Panagia N., 2008, ApJ, 676, 781

Wild V., Walcher C. J., Johansson P. H., Tresse L., Charlot S., Pollo A., Le Fèvre O., de Ravel L., 2009, MNRAS, 395, 144

Willmer C. N. A. et al., 2006, ApJ, 647, 853

Wu P.-F., Gal R. R., Lemaux B. C., Kocevski D. D., Lubin L. M., Rumbaugh N., Squires G. K., 2014, ApJ, 792, 16

Wuyts S. et al., 2011, ApJ, 742, 96

Yeh S. C. C., Matzner C. D., 2012, ApJ, 757, 108

Zabludoff A. I., Zaritsky D., Lin H., Tucker D., Hashimoto Y., Shectman S. A., Oemler A., Kirshner R. P., 1996, ApJ, 466, 104

This paper has been typeset from a $\mathrm{T}_{\mathrm{E}} \mathrm{X} / \mathrm{L} \mathrm{T}_{\mathrm{E}} \mathrm{X}$ file prepared by the author. 